\title{
Cold Dark Matter in non-Standard Cosmologies, PAMELA, ATIC AND FERMi LAT
}

\author{
C. Pallis \\ Department of Physics, University of Patras, \\ GR-265 00 Patras, GREECE \\ e-mail address: kpallis@auth.gr
}

\begin{abstract}
We consider two non-standard cosmological scenaria according to which the universe is reheated to a low reheating temperature after the late decay of a scalar field or is dominated by the kinetic energy of a quintessence field in the context of a tracking quintessential model. In both cases, we calculate the relic density of the Weakly Interacting Massive Particles (WIMPs) and show that it can be enhanced with respect to its value in the standard cosmology. By adjusting the parameters of the low reheating or the quintessential scenario, the cold dark matter abundance in the universe can become compatible with large values for the annihilation cross section times the velocity of the WIMPs. Using these values and assuming that the WIMPs annihilate predominantly to $e^{+} e^{-}, \mu^{+} \mu^{-}$or $\tau^{+} \tau^{-}$, we calculate the induced fluxes of $e^{ \pm}$cosmic rays and fit the current data of PAMELA and ATIC or Fermi LAT. We achieve rather good fits especially to PAMELA and Fermi-LAT data in conjunction with a marginal fulfillment of the restriction arising from cosmic microwave background, provided that the WIMPs annihilate predominantly to $\mu^{+} \mu^{-}$. In both non-standard scenaria the required transition temperature to the conventional radiation dominated era turns out to be lower than about $0.7 \mathrm{GeV}$. In the case of the low reheating, an appreciable non-thermal contribution to the WIMP relic density is also necessitated.
\end{abstract}

Keywords: Cosmology, Dark Matter

PACS coDEs: $98.80 . \mathrm{Cq}, 95.35 .+\mathrm{d}$

Published in Nucl. Phys. B831, 217 (2010) 


\section{Contents}

1. INTRODUCTION 1

2. Non-Standard Cosmological Scenaria 3

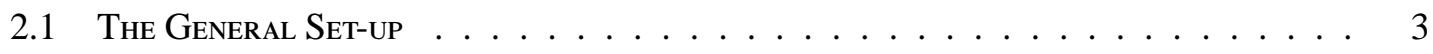

2.2 The Low Reheating Scenario $\ldots \ldots \ldots \ldots \ldots \ldots$

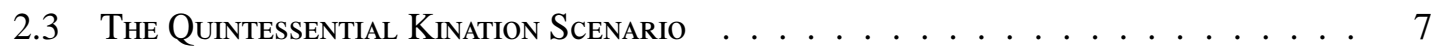

3. The WIMP Relic Density 11

3.1 The BoltzMann Equation . . . . . . . . . . . . . . . . . . . . . . . . 11

3.2 The ENHANCEMENT of $\Omega_{\chi} h^{2} \ldots \ldots \ldots \ldots \ldots \ldots \ldots \ldots$

4. PAMELA, ATIC and Fermi-LAT Anomalies 16

4.1 Cosmic RaYs from AnNiHILATION of WIMPs $\ldots \ldots \ldots \ldots \ldots \ldots$

4.2 Fitting the PAMELA and ATIC or Fermi-LAT Data $\ldots \ldots \ldots \ldots$

5. Restrictions in the $m_{\chi}-\langle\sigma v\rangle$ Plane 20

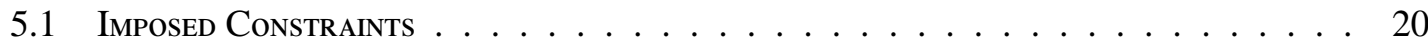

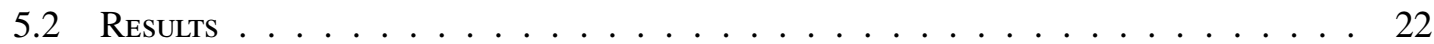

6. Conclusions 25

\section{INTRODUCTION}

The accurate determination of cosmological parameters by up-to-date observations, most notably by the Wilkinson Microwave Anisotropy Probe (WMAP) [1, 2], establishes a quite extensive and convincing evidence for the constitution of the present universe by an enigmatic component called Cold Dark Matter (CDM) with abundance, $\Omega_{\mathrm{CDM}} h^{2}$, in the following range:

$$
\Omega_{\mathrm{CDM}} h^{2}=0.1099 \pm 0.0124
$$

at 95\% confidence level (c.1.). Natural candidates [3] to account for the CDM are [4] the weakly interacting massive particles (WIMPs, hereafter denoted as $\chi$ ) with prominent representative (for other WIMPs, see Ref. [5, 6]) the lightest neutralino [7] which turns out to be the lightest supersymmetric (SUSY) particle (LSP) in a sizeable fraction of the parameter space of the SUSY models and therefore, stable under the assumption of the $R$-parity conservation. In view of Eq. (1.1), the relic density of $\chi$ 's, $\Omega_{\chi} h^{2}$, is to satisfy a very narrow range of values:

$$
\text { (a) } 0.097 \lesssim \Omega_{\chi} h^{2} \text { and (b) } \Omega_{\chi} h^{2} \lesssim 0.12 \text {, }
$$

with the lower bound being valid under the assumption that CDM is entirely composed by $\chi$ 's.

The calculation of $\Omega_{\chi} h^{2}$ crucially depends [8] on the adopted assumption about the dominant component of the universe during the decoupling of WIMPs. The usual assumption is that this 
occurs during the radiation dominated (RD) epoch which commences after the primordial inflation. However, our ignorance about the universal history before Big Bang nucleosynthesis (BBN) allows for other possibilities. E.g., the presence of a scalar field, which dominates the budget of the universal energy density through its potential $[8,9,10,11,12,13]$ or kinetic $[8,14,15,16]$ energy density, can enhance significantly $\Omega_{\chi} h^{2}$ with respect to (w.r.t) its value in the standard cosmology (SC). In the first case, the scalar field can generate an episode of low reheating which can be accompanied by thermal and/or non-thermal production of $\chi$ 's. In the second case, a kination dominated (KD) epoch [17], which may be embedded [16, 18, 19, 20, 21] in a quintessential framework, can arise. As a bonus, in the latter case, the problem of the second major component of the present universe, called Dark Energy (DE) can be addressed - for reviews see, e.g., Ref. [22].

The aforementioned enhancements of $\Omega_{\chi} h^{2}$ have attracted much attention recently [23] since they assist us to interpret, through WIMP annihilation in the galaxy and consistently with Eq. (1.1), the reported $[24,25]$ excess on the positron $\left(e^{+}\right)$and/or electron $\left(e^{-}\right)$cosmic-ray (CR) flux, without invoking any pole effect [26], ad-hoc boost factor [27] or other astrophysical sources [28]. In particular, PAMELA experiment has reported [24] (confirming previous experiments [29]) an unexpected rise of $e^{+}$flux fraction for values of the $e^{+}$energy, $E_{e^{+}}$, in the range $(10-100) \mathrm{GeV}$, in contrast to the power-law falling background. Moreover, data by the ATIC experiment [25] shows an excess in the total $e^{+}$and $e^{-}$flux for $300 \leq E_{e^{+}} / \mathrm{GeV} \leq 800$. On the other hand, the very recently released data from Fermi LAT indicates [30] smaller fluxes than the ATIC data in the same range of energies. Nevertheless, we consider (separately) both latter data in our study.

In this paper we reconsider the increase of $\Omega_{\chi} h^{2}$ within a low reheating scenario (LRS) or a quintessential kination scenario (QKS) in light of the experimental results above. Namely, we recall (Sec. 2) comparatively the salient features of the two non-standard scenaria, solving numerically the relevant system of equations, reviewing the cosmological dynamics and imposing a number of observational constraints. Particularly, in the LRS we consider the late decay of a massive field which reheats the universe to a low reheating temperature. In the QKS, we consider the recently implemented [21] generation of a KD era (associated with an oscillatory evolution of the quintessence field) in the context of tracking quintessential model with a Hubble-induced mass term for the quintessence field. We then (Sec. 3) investigate the enhancement of $\Omega_{\chi} h^{2}$ w.r.t its value in SC within these non-standard scenaria. We show that the increase of $\Omega_{\chi} h^{2}$ depends on (i) the reheat temperature and the number of $\chi$ 's produced per decay and unit mass of the decaying field, in the case of LRS, and (ii) the proximity between the freeze-out temperature and the temperature where the evolution of the quintessence develops extrema, in the case of QKS. We also present (Sec. 4) the energy spectra of the $e^{ \pm}-\mathrm{CR}$, assuming that $\chi$ 's annihilate into $e^{+} e^{-}, \mu^{+} \mu^{-}$ or $\tau^{+} \tau^{-}$and adopting an isothermal halo profile [31]. Although Cosmic Microwave Background (CMB) $[32,33,34]$ tightly constrain the relevant parameters, we achieve rather satisfactory fittings especially to the combination of PAMELA and Fermi-LAT $e^{ \pm}$-CR data and for the case where $\chi$ 's annihilate into $\mu^{+} \mu^{-}$(Sec. 5). Fulfilment of Eq. (1.2) is also possible by appropriately adjusting the parameters of the LRS or QKS. We end up with our conclusions in Sec. 6.

Throughout the text, brackets are used by applying disjunctive correspondence, the subscript or superscript 0 is referred to present-day values (except for the coefficient $\bar{V}_{0}$ ) and $\log [\ln ]$ stands for logarithm with basis $10[e]$. Besides Sec. 4, natural units for the Planck's constant, Boltzmann's constant and the velocity of light $\left(\hbar=c=k_{\mathrm{B}}=1\right)$ are assumed. 


\section{Non-Standard Cosmological Scenaria}

In this section we present comparatively the main features of the two non-standard cosmological scenaria under consideration. Namely, in Sec. 2.1 we expose the basic assumptions of each scenario with reference to the SC and introduce notation. Sec. 2.2 and Sec. 2.3 are devoted to a review of the LRS and QKS respectively. Despite the fact that the displayed scenaria have been already analyzed in Ref. [11, 21] we prefer to briefly recall and update our results for completeness and clarity.

\subsection{The General Set-up}

According to SC, primordial inflation is followed by a RD era. The $\chi$ species (i) are produced through thermal scatterings in the plasma, (ii) reach chemical equilibrium with plasma and (iii) decouple from the cosmic fluid at a temperature $T_{\mathrm{F}} \sim(10-20) \mathrm{GeV}$ during the RD era. The assumptions above fix the form of the relevant Boltzmann equation, the required strength of the $\chi$ interactions for thermal production (TP) and lead to an isentropic cosmological evolution during the $\chi$ decoupling: The Hubble parameter is $H \propto T^{2}$ with temperature $T \propto R^{-1}$ where $R$ is the scale factor of the universe. In this context, the $\Omega_{\chi} h^{2}$ calculation depends only on two parameters: The $\chi$ mass, $m_{\chi}$ and the thermal-averaged cross section of $\chi$ times velocity, $\langle\sigma v\rangle-$ see Table 1 . Although $\langle\sigma v\rangle$ can be derived from $m_{\chi}$ and the residual (s)particle spectrum once a low energy theory is adopted (see, e.g., Ref. [15]), we treat $m_{\chi}$ and $\langle\sigma v\rangle$ as unrelated input parameters in order to keep our approach as general as possible (see, e.g., Ref. [16, 31]). Also, to be in harmony with the assumptions employed in the derivation of the restrictions mentioned in Sec. 5.1, we consider throughout constant $\langle\sigma v\rangle$ 's, i.e., independent of $T$.

The modern cosmo-particles theories, however, are abundant in scalar massive particles (moduli) which can decay out of equilibrium when $H$ becomes equal to their mass creating episodes of reheating. In the LRS, we assume that such a scalar particle $\phi$, with mass $m_{\phi}$, decays with a rate $\Gamma_{\phi}$ into radiation, producing an average number $N_{\chi}$ of $\chi$ 's. The key point in this case is that the reheating process is not instantaneous $[9,11]$. During its realization, the maximal temperature, $T_{\max }$, is much larger than the so-called reheat temperature, $T_{\mathrm{RH}}$, which can be taken to be lower than $T_{\mathrm{F}}$. Also, for $T>T_{\mathrm{RH}}, H \propto T^{4}$ with $T \propto R^{-3 / 8}$ and an entropy production occurs (in contrast with the SC). The $\chi$ species (i') decouple during the decaying- $\phi$ dominated era (ii') do or do not reach chemical equilibrium with the thermal bath (iii') are produced by thermal scatterings and directly by the $\phi$ decay (which naturally arises even without direct coupling [9]). As a consequence, the $\Omega_{\chi} h^{2}$ calculation depends also on $T_{\mathrm{RH}}, m_{\phi}$ and $N_{\chi}$ - see Table 1 .

Another role that a scalar field could play when it does not couple to matter (contrary to $\phi$ ) is this of quintessence. In our QKS, such a scalar field $q$ (not to be confused with the deceleration parameter [2]) is supposed to roll down its inverse power-law potential (with exponent $a$ and a mass scale $M$ ) supplemented with a Hubble-induced mass term (with coefficient $b$ ) motivated mainly by non-canonical Kähler potential $[20,35]$ - c.f. Ref. [36]. A mild tuning of $b$ and of the initial conditions at an initial temperature $T_{\mathrm{I}}-H_{\mathrm{I}}=H\left(T_{\mathrm{I}}\right)$ and $q_{\mathrm{I}}=q\left(T_{\mathrm{I}}\right)$ - ensures the coexistence of an early modified KD phase with the tracking $[37,38]$ solutions and offers the desirable property of the insensitivity to the initial conditions [20,21]. Since the $q$ kinetic energy, which decreases as $T^{6}$ (except for isolated points) dominates we get $H \propto T^{3}$ with $T \propto R^{-1}$. If the $\chi$-decoupling occurs during this KD phase - the assumptions (i) and (ii) are maintained - the $\Omega_{\chi} h^{2}$ calculation depends also on $a, b, M, H_{\mathrm{I}}, T_{\mathrm{I}}$ and $q_{\mathrm{I}}$ in this scenario - see Table 1. 


\begin{tabular}{|c|c|c|}
\hline $\mathbf{S C}$ & LRS & QKS \\
\hline \hline $\bar{\rho}_{q}=\bar{\rho}_{\phi}=0$ & $\bar{\rho}_{\phi_{\mathrm{I}}} \gg \bar{\rho}_{\mathrm{RI}}, \bar{\rho}_{q}=0$ & $\bar{\rho}_{q_{\mathrm{I}}} \gg \bar{\rho}_{\mathrm{RI}}, \bar{\rho}_{\phi}=0$ \\
$H \propto T^{2}$ & $H \propto T^{4}$ & $H \propto T^{3}$ \\
$T \propto R^{-1}$ & $T \propto R^{-3 / 8}$ & $T \propto R^{-1}$ \\
$s R^{3}=\mathrm{cst}$ & $s R^{3} \neq \mathrm{cst}$ & $s R^{3}=\mathrm{cst}$ \\
$N_{\chi}=0$ & $N_{\chi} \neq 0$ & $N_{\chi}=0$ \\
\hline \multicolumn{2}{|c|}{ FREE PARAMETERS OF THE $\Omega_{\chi} h^{2}$ CALCULATION } \\
\hline$m_{\chi},\langle\sigma v\rangle$ & $m_{\chi},\langle\sigma v\rangle$, & $m_{\chi},\langle\sigma v\rangle$, \\
\multicolumn{2}{|c|}{$T_{\mathrm{RH}}, m_{\phi}, N_{\chi}$} & $a, b, M, H_{\mathrm{I}}, q_{\mathrm{I}}, T_{\mathrm{I}}$ \\
\hline
\end{tabular}

TABLE 1: Comparing the $S C$ with the LRS and the QKS (the various symbols are explained in Sec. 2.1, the subscript I is referred to the onset of each scenario and "cst" stands for "constant").

In the two non-standard scenaria under consideration, $H$ is given by

$$
H^{2}=\frac{1}{3 m_{\mathrm{P}}^{2}} \begin{cases}\left(\rho_{\phi}+\rho_{\chi}+\rho_{\mathrm{R}}\right) & \text { for the LRS, } \\ \left(\rho_{q}+\rho_{\mathrm{R}}+\rho_{\mathrm{M}}\right) & \text { for the QKS, }\end{cases}
$$

where $\rho_{i}$ with $i=\phi, q$ and $\chi$ is the energy density of $\phi, q$ and $\chi$ respectively and $m_{\mathrm{P}}=M_{\mathrm{P}} / \sqrt{8 \pi}$ where $M_{\mathrm{P}}=1.22 \cdot 10^{19} \mathrm{GeV}$ is the Planck mass. The energy density of radiation, $\rho_{\mathrm{R}}$, and the entropy density, $s$, can be evaluated as a function of $T$, whilst the energy density of matter, $\rho_{\mathrm{M}}$, with reference to its present-day value:

$$
\rho_{\mathrm{R}}=\frac{\pi^{2}}{30} g_{\rho *} T^{4}, s=\frac{2 \pi^{2}}{45} g_{s *} T^{3} \text { and } \rho_{\mathrm{M}} R^{3}=\rho_{\mathrm{M} 0} R_{0}^{3}
$$

where $g_{\rho *}(T)\left[g_{s *}(T)\right]$ is the energy [entropy] effective number of degrees of freedom at temperature $T$. Their precise numerical values are evaluated by using the tables included in public packages [39] and assuming the particle spectrum of the Minimal Supersymmetric Standard Model.

The initial value of $H, H_{\mathrm{I}}$, in both non-standard scenaria can be restricted, assuming that a primordial phase of inflation (driven by a scalar field different from $\phi$ or $q$, in general) is responsible for the generation of the power spectrum of the curvature scalar $P_{\mathrm{s}}$ and tensor $P_{\mathrm{t}}$ perturbations. Indeed, imposing the conservative restriction $r=P_{\mathrm{t}} / P_{\mathrm{s}} \lesssim 1$ and using the observational [1] normalization of $P_{\mathrm{s}}$, an upper bound on $H_{\mathrm{I}}$ can be found as follows:

$$
H_{\mathrm{I}} \lesssim \frac{\pi}{\sqrt{2}} m_{\mathrm{P}} P_{\mathrm{s} *}^{1 / 2} \Rightarrow H_{\mathrm{I}} \lesssim 2.65 \cdot 10^{14} \mathrm{GeV}
$$

where $*$ means that $P_{\mathrm{s} *}$ is measured at the pivot scale $k_{*}=0.002 / \mathrm{Mpc}$.

Let us, finally, introduce a set of normalized quantities which simplify significantly the relevant formulas. In particular we define

$$
\begin{aligned}
& \bar{\rho}_{i}=\rho_{i} / \rho_{\mathrm{c} 0}, \text { with } i=\mathrm{R}, \mathrm{M}, \phi \text { and } q, \bar{J}=J / \rho_{\mathrm{c} 0}^{3 / 4} \text { with } J=n_{\chi}, n_{\chi}^{\mathrm{eq}} \text { and } n_{\phi}, \\
& \bar{m}_{i}=m_{i} / \rho_{\mathrm{c} 0}^{1 / 4} \text { with } i=\chi \text { and } \phi, \bar{J}=J / H_{0} \text { with } J=H \text { and } \Gamma_{\phi} \text { and } \overline{\langle\sigma v\rangle}=\sqrt{3} m_{\mathrm{P}} \rho_{\mathrm{c} 0}^{1 / 4}\langle\sigma v\rangle
\end{aligned}
$$

where $n_{i}$ with $i=\phi$ and $\chi$ is the number density of $\chi$ and $\phi$ respectively. Note that $\rho_{\chi}=m_{\chi} n_{\chi}$ and $\rho_{\phi}=m_{\phi} \Delta_{\phi} n_{\phi}$ where $\Delta_{\phi}=\left(m_{\phi}-N_{\chi} m_{\chi}\right) / m_{\phi}$. In our numerical calculation, we use the values:

$$
\rho_{\mathrm{c} 0} \simeq 8.1 \cdot 10^{-47} h^{2} \mathrm{GeV}^{4}, \text { with } h=0.72, \bar{\rho}_{\mathrm{M} 0}=0.26 \text { and } T_{0}=2.35 \cdot 10^{-13} \mathrm{GeV} \text {. }
$$

We have also $H_{0}=2.13 \cdot 10^{-42} h \mathrm{GeV}$ and from Eq. (2.2) we get $\bar{\rho}_{\mathrm{R} 0}=8.04 \cdot 10^{-5}$. 


\subsection{The Low Reheating Scenario}

We below (Sec. 2.2.1) present the system of equations which governs the cosmological evolution in the LRS, summarize (Sec. 2.2.2) the various observational restrictions that have to be imposed and sketch (Sec. 2.2.3) the basics of the relevant dynamics.

2.2.1 Relevant Equations. Under the assumption that the decay products of $\phi$ are rapidly thermalized (see below) the energy densities $\rho_{\phi}$ and $\rho_{\mathrm{R}}$ obey the following Boltzmann equations

$$
\begin{aligned}
& \dot{\rho}_{\phi}+3 H \rho_{\phi}+\Gamma_{\phi} \rho_{\phi}=0, \\
& \dot{\rho}_{\mathrm{R}}+4 H \rho_{\mathrm{R}}-\Gamma_{\phi} \rho_{\phi}-2 m_{\chi}\langle\sigma v\rangle\left(n_{\chi}^{2}-n_{\chi}^{\mathrm{eq} 2}\right)=0,
\end{aligned}
$$

where dot stands for derivative w.r.t the cosmic time, $t$ and $n_{\chi}^{\mathrm{eq}}$ is the equilibrium number density of $\chi$, which obeys the Maxwell-Boltzmann statistics:

$$
n_{\chi}^{\mathrm{eq}}(x)=\frac{g}{(2 \pi)^{3 / 2}} m_{\chi}^{3} x^{3 / 2} e^{-1 / x} P_{2}\left(\frac{1}{x}\right), \text { where } x=\frac{T}{m_{\chi}} \text { and } P_{n}(z)=1+\frac{\left(4 n^{2}-1\right)}{8 z}
$$

is obtained by expanding the modified Bessel function of the 2nd kind of order $n$ for $x \ll 1$. Assuming that $\chi$ 's are Majorana fermions, we set $g=2$ for their number of degrees of freedom. Note that although in our numerical program Eqs. (2.6a) and (2.6b) are resolved together with Eq. (3.2) - see Sec. 3.1-, we here prefer to present just the two first equations since the influence of $n_{\chi}$ to the dynamics of reheating via the last term of the left hand side in Eq. (2.6b) is in general negligible. Moreover, $\Gamma_{\phi}$ can be replaced by $T_{\mathrm{RH}}$ through the relation [11]:

$$
\Gamma_{\phi}=5 \sqrt{\frac{\pi^{3} g_{\rho *}\left(T_{\mathrm{RH}}\right)}{45}} \frac{T_{\mathrm{RH}}^{2}}{M_{\mathrm{P}}}=\sqrt{\frac{5 \pi^{2} g_{\rho *}\left(T_{\mathrm{RH}}\right)}{72}} \frac{T_{\mathrm{RH}}^{2}}{m_{\mathrm{P}}} .
$$

Note that the adopted prefactor, which is slightly different than those used in the bibliography $[10,12]$, assists us to approach accurately the numerical solution of $\rho_{\phi}\left(T_{\mathrm{RH}}\right)=\rho_{\mathrm{R}}\left(T_{\mathrm{RH}}\right)$.

The numerical integration of Eqs. (2.6a) and (2.6b) is facilitated by absorbing the dilution terms. To this end, we find it convenient to define the following variables $[10,11]$ :

$$
f_{\phi}=\bar{\rho}_{\phi} R^{3}, f_{\mathrm{R}}=\bar{\rho}_{\mathrm{R}} R^{4}, f_{\chi}=\bar{n}_{\chi} R^{3} \text { and } f_{\chi}^{\mathrm{eq}}=\bar{n}_{\chi}^{\mathrm{eq}} R^{3}
$$

and convert the time derivatives to derivatives w.r.t the logarithmic time [11]:

$$
\tilde{\tau}=\ln \left(R / R_{\mathrm{I}}\right) \Rightarrow R^{\prime}=R \text { and } R=R_{\mathrm{I}} e^{\tilde{\tau}}
$$

where prime in this section denotes derivation w.r.t $\tilde{\tau}$ and the value of $R_{\mathrm{I}}$ in this definition can be conveniently selected so as the resolution of the system is numerically stable. After realize the modifications above, Eqs. (2.6a) and (2.6b) become:

$$
\text { (a) } \bar{H} f_{\phi}^{\prime}=-\bar{\Gamma}_{\phi} f_{\phi} \text { and (b) } \bar{H} R^{2} f_{\mathrm{R}}^{\prime}=\bar{\Gamma}_{\phi} f_{\phi} R^{3}+2 \bar{m}_{\chi} \overline{\langle\sigma v\rangle}\left(f_{\chi}^{2}-f_{\chi}^{\mathrm{eq} 2}\right) \text {, }
$$

where $H$ and $T$ can be expressed correspondingly, in terms of the variables in Eq. (2.9), as follows:

$$
\text { (a) } \bar{H}=R^{-3 / 2} \sqrt{f_{\phi}+f_{\mathrm{R}} / R} \text { and (b) } T=\left(\frac{30 f_{\mathrm{R}}}{\pi^{2} g_{\rho *} R^{4}} \rho_{\mathrm{c} 0}\right)^{1 / 4} \text {. }
$$


The system of Eq. (2.11) can be solved from 0 to $\tilde{\tau}_{f} \sim 50$, imposing the following initial conditions (recall that the subscript I is referred to quantities defined at $\tilde{\tau}=0$ ):

$$
H_{\mathrm{I}}=m_{\phi} \Rightarrow \bar{\rho}_{\phi_{\mathrm{I}}}=m_{\phi}^{2} / H_{0}^{2} \text { and } \bar{\rho}_{\mathrm{RI}}=\bar{\rho}_{\chi_{\mathrm{I}}}=0 .
$$

However, the results on $\Omega_{\chi} h^{2}$ do not depend on the explicit value of $\bar{\rho}_{\phi_{\mathrm{I}}}$ as long as $T_{\mathrm{RH}}<T_{\mathrm{F}}<T_{\max }$, and are invariant $[11,12]$ for fixed $N_{\chi} m_{\phi}^{-1}$ (and $T_{\phi}, m_{\chi},\langle\sigma v\rangle$ ). Therefore, for presentation purposes, it is convenient to define the following quantity [12]:

$$
c_{\chi \phi}=N_{\chi} \frac{100 \mathrm{TeV}}{m_{\phi}}
$$

2.2.2 ImPosed ReQuiRements. We impose on the LRS the following requirements:

- The BBN Constraint. The presence of $\rho_{\phi}$ should not jeopardize the successful predictions of $\mathrm{BBN}$ which commences at about $T_{\mathrm{BBN}}=1 \mathrm{MeV}$ [40]. Namely, we require:

$$
T_{\mathrm{RH}} \geq 1 \mathrm{MeV} \text { (95\% c.l.). }
$$

Given that $\phi$ decays mostly through gravitational interactions, $\Gamma_{\phi}$ and consequently $T_{\mathrm{RH}}-$ see Eq. (2.8) - are highly suppressed. Therefore [12] fulfilment of BBN constraint with more or less natural coupling constants requires $m_{\phi} \geq 100 \mathrm{TeV}$.

- Constraints on the range of $m_{\phi}$. Eq. (2.3) assists us to impose an upper bound on $m_{\phi}$ due to our initial condition in Eq. (2.13). On the other hand, $m_{\phi}$ can be bounded from below, too, demanding the decay of $\phi$ to a pair of $\chi$ 's (with mass $m_{\chi}$ ) to be kinematically allowed. All in all we require:

$$
2 m_{\chi} \leq m_{\phi} \lesssim 2.65 \cdot 10^{14} \mathrm{GeV} .
$$

Note that the upper bound of Eq. (2.16) assures also the rapid thermalization of the $\phi$-decay products. Indeed, the latter condition, which is crucial for Eqs. (2.6a) and (2.6b) to be applicable, is satisfied [41] for $m_{\phi} \lesssim 8 \cdot 10^{14} \mathrm{GeV}$.

- Constraint on the range of $N_{\chi}$. Depending on the coupling between $\phi$ and $\chi$ in a specific theory, a variety of $N_{\chi}$ 's is possible $[9,12,42]$. In our approach we conservatively set the upper bound $N_{\chi} \leq 1$.

Let us finally mention that, on quite general ground, any modulus $\phi$ has an unsuppressed coupling to gravitino, $\widetilde{G}$. Possible decay of $\phi$ to $\widetilde{G}$ creates the co-called moduli-induced $\widetilde{G}$ problem [42]. To avoid these complications, we are obliged to assume that the masses of $\widetilde{G}$ and $\phi$ are of the same order of magnitude.

2.2.3 Dynamics of Reheating. The cosmological evolution of the various quantities involved in the LRS as a function of $\tilde{\tau}$ is illustrated in Fig. 1 for $m_{\phi}=100 \mathrm{TeV}$ and $\left(N_{\chi}, T_{\mathrm{RH}}\right)=(1,0.5 \mathrm{GeV}$ ) (gray lines) or $\left(N_{\chi}, T_{\mathrm{RH}}\right)=\left(7.5 \cdot 10^{-5}, 1 \mathrm{MeV}\right)$ (light gray lines). In particular, we design $\log \bar{\rho}_{i}$ with $i=\phi$ (solid lines) and $i=\mathrm{R}$ (dashed lines) $[T]$ versus $\tilde{\tau}$ in Fig. 2-(a) [Fig. 2-(b)]. The quantities $\bar{\rho}_{i}$ with $i=\phi$ and $\mathrm{R}[T]$ are computed by substituting the numerical solution of Eq. (2.11) into Eq. (2.9) [Eq. (2.12b)]. From Fig. 1 we can understand the dynamics of the universe during the two distinct phases $[10,11]$ : 


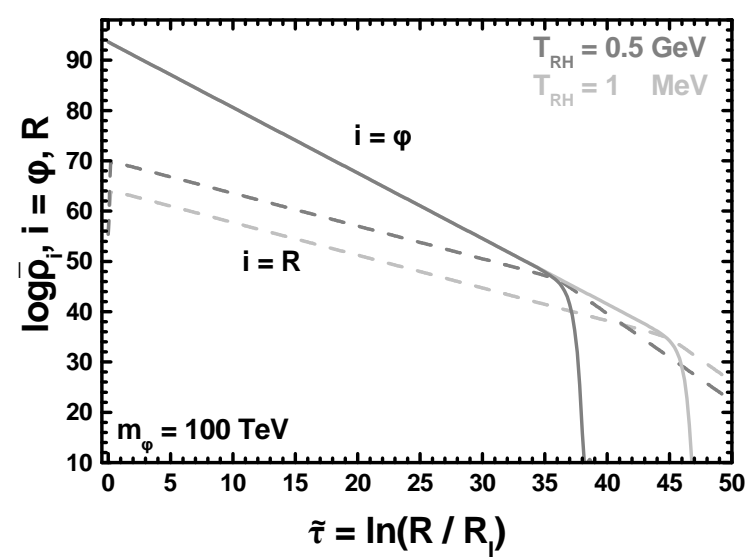

(a)

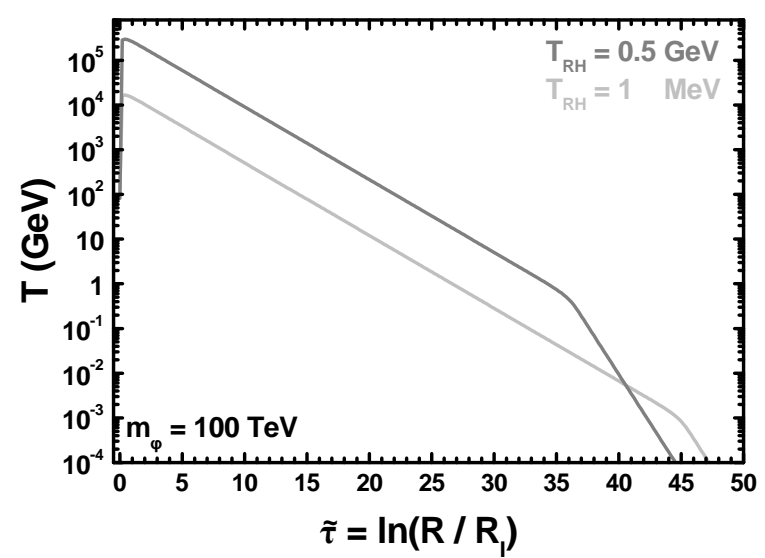

(b)

Figure 1: The evolution of (a) $\log \bar{\rho}_{i}$ with $i=\phi$ (solid lines) and $R$ (dashed lines) and (b) $T$ as a function of $\tilde{\tau}=\ln \left(R / R_{\mathrm{I}}\right.$ ) for $m_{\phi}=100 \mathrm{TeV}$ and $\left(N_{\chi}, T_{\mathrm{RH}}\right)=(1,0.5 \mathrm{GeV})$ (gray lines) or $\left(N_{\chi}, T_{\mathrm{RH}}\right)=\left(7.5 \cdot 10^{-5}, 1 \mathrm{MeV}\right)$ (light gray lines). In both cases, we take $\Omega_{\chi} h^{2}=0.11$ for $m_{\chi}=0.5 \mathrm{TeV}$ and $\langle\sigma v\rangle=3 \cdot 10^{-7} \mathrm{GeV}^{-2}$.

- For $T \gg T_{\mathrm{RH}}$, we have $\rho_{\phi} \gg \rho_{\mathrm{R}}$. Consequently, inserting $\bar{H} \simeq \sqrt{\bar{\rho}_{\phi}}$ into Eq. (2.11) we extract:

$$
\text { (a) } \bar{\rho}_{\phi}=\bar{\rho}_{\phi_{\mathrm{I}}} e^{-3 \tilde{\tau}} \text { and (b) } \bar{\rho}_{\mathrm{R}}=\frac{2}{5} \bar{\Gamma}_{\phi} \bar{\rho}_{\phi_{\mathrm{I}}}^{1 / 2}\left(e^{-3 \tilde{\tau} / 2}-e^{-4 \tilde{\tau}}\right) \text {. }
$$

The function $\bar{\rho}_{\mathrm{R}}(\tilde{\tau})$ reaches at $\tilde{\tau}_{\max } \simeq \ln (1.48)=0.39$ a maximum value $\bar{\rho}_{\text {Rmax }} \simeq 0.14 \sqrt{\bar{\rho}_{\phi_{\mathrm{I}}}} \bar{\Gamma}_{\phi}$ corresponding to a $T=T_{\max }$ derived through Eq. (2.2). The completion of the reheating is realized at $\tilde{\tau}=\tilde{\tau}_{R H}$, such that:

$$
\rho_{\mathrm{R}}\left(\tilde{\tau}_{\mathrm{RH}}\right)=\rho_{\phi}\left(\tilde{\tau}_{\mathrm{RH}}\right) \Rightarrow \tilde{\tau}_{\mathrm{RH}} \simeq-\frac{2}{3} \ln \frac{2}{5} \bar{\Gamma}_{\phi} \bar{\rho}_{\phi_{\mathrm{I}}}^{-1 / 2} .
$$

where a corner is observed on the curves of Fig. 1-(b).

- For $T<T_{\mathrm{RH}}$, we get $\rho_{\mathrm{R}} \gg \rho_{\phi}$, and so, $\bar{H} \simeq \sqrt{\bar{\rho}_{\mathrm{R}}}$. Plugging it into Eq. (2.11) we can obtain approximately the following expressions:

$$
\bar{\rho}_{\phi}=\bar{\rho}_{\phi}\left(\tilde{\tau}_{\mathrm{RH}}\right) \exp \left(-3\left(\tilde{\tau}-\tilde{\tau}_{\mathrm{RH}}\right)-\frac{5}{4}\left(e^{2\left(\tilde{\tau}-\tilde{\tau}_{R H}\right)}-1\right)\right) \text { and } \bar{\rho}_{\mathrm{R}}=\bar{\rho}_{\mathrm{R}}\left(\tilde{\tau}_{\mathrm{RH}}\right) e^{-4\left(\tilde{\tau}-\tilde{\tau}_{\mathrm{RH}}\right)} .
$$

\subsection{The Quintessential Kination Scenario}

We present below (Sec. 2.3.1) the equations which govern the cosmological evolution in the QKS and then enumerate (Sec. 2.3.2) the various observational restrictions that have to be imposed. We also highlight the $q$ dynamics (Sec. 2.3.3) and describe the allowed parameter space (Sec. 2.3.4).

2.3.1 Relevant Equations. Under the assumption that $q$ is spatially homogeneous, it obeys the following Klein-Gordon equation:

$$
\ddot{q}+3 H \dot{q}+V_{, q}=0 \text {, where } V=V_{a}+V_{b} \text { with } V_{a}=\frac{M^{4+a}}{q^{a}} \text { and } V_{b}=\frac{b}{2} H^{2} q^{2},
$$

is the adopted potential for the field $q$ with $M$ a mass-scale and , $q$ stands for derivative w.r.t $q$. In our approach $V_{b}$ is present throughout the cosmological evolution of $q$. The induced coupling 
between $q$ and CDM during the matter dominated era is too suppressed to have any observational consequence. Nonetheless, we have checked that our results remain intact even if we switch off this term after the onset of the matter domination. The numerical integration of Eq. (2.20) is facilitated by converting the time derivatives to derivatives w.r.t the logarithmic time [16] which is defined as a function of the redshift $z$ :

$$
\tau=\ln \left(R / R_{0}\right)=-\ln (1+z) .
$$

Changing the differentiation and introducing the following quantities:

$$
\bar{V}_{a}=V_{a} / \rho_{\mathrm{c} 0}, \quad f_{q}=\dot{q} R^{3} / \sqrt{\rho_{\mathrm{c} 0}} \text { and } \bar{q}=q / \sqrt{3} m_{\mathrm{P}},
$$

Eq. (2.20) turns out to be equivalent to the system of two first-order equations:

$$
\begin{gathered}
f_{q}=\bar{H} \bar{q}^{\prime} R^{3} \quad \text { and } \bar{H} f_{q}^{\prime} / R^{3}+b \bar{H}^{2} \bar{q}+b \bar{H} \bar{H}_{, \bar{q}} \bar{q}^{2}+\bar{V}_{a, \bar{q}}=0, \\
\text { where } \quad \bar{H}^{2}=\frac{1}{1-b \bar{q}^{2} / 2}\left(\frac{1}{2} f_{q}^{2} / R^{6}+\bar{V}_{a}+\bar{\rho}_{\mathrm{R}}+\bar{\rho}_{\mathrm{M}}\right),
\end{gathered}
$$

prime in this section denotes derivative w.r.t. $\tau$ and $M$ can be found from the dimensionless quantities as follows:

$$
M=\left(\left(\sqrt{3} m_{\mathrm{P}}\right)^{a} \bar{V}_{0} \rho_{\mathrm{c} 0}\right)^{1 /(4+a)} \text { with } \bar{V}_{a}=\bar{V}_{0} / \bar{q}^{a} .
$$

Eq. (2.23) can be resolved numerically if two initial conditions are specified at an initial $\tau, \tau_{I}$ corresponding to a temperature $T_{\mathrm{I}}$, which is defined as the maximal $T$ after the end of primordial inflation, assuming instantaneous reheating. We take $\bar{q}\left(\tau_{\mathrm{I}}\right)=10^{-2}$ throughout our investigation, without any loss of generality (see below) and let as free parameter $\bar{H}_{\mathrm{I}}=\bar{H}\left(T_{\mathrm{I}}\right)$.

2.3.2 Imposed Requirements. Our QKS can be [21] consistent with the following restrictions:

- Constraints on the initial conditions. We focus on the initial conditions which assure a complete initial domination of kination consistently with Eq. (2.3), i.e.,

$$
\begin{aligned}
& \text { (a) } \Omega_{q}^{\mathrm{I}}=\Omega_{q}\left(T_{\mathrm{I}}\right)=1 \text { and (b) } \bar{H}_{\mathrm{I}} \lesssim 1.72 \cdot 10^{56} \\
& \text { with } \Omega_{q}=\rho_{q} /\left(\rho_{q}+\rho_{\mathrm{R}}+\rho_{\mathrm{M}}\right) \text { where } \rho_{q}=\dot{q} / 2+V \text {. }
\end{aligned}
$$

- The BBN Constraint. The presence of $\rho_{q}$ has not to spoil the successful predictions of BBN which commences at about $\tau_{\mathrm{BBN}}=-22.5\left(T_{\mathrm{BBN}}=1 \mathrm{MeV}\right)$ [40]. Namely, we require:

$$
\Omega_{q}^{\mathrm{BBN}}=\Omega_{q}\left(\tau_{\mathrm{BBN}}\right) \leq 0.21(95 \% \text { c.l. })
$$

where 0.21 corresponds to additional effective neutrinos species $\delta N_{v}<1.6$ [40].

- DE-Density and Coincidence Constraint. These two constraints can be addressed if (i) the present value of $\rho_{q}, \rho_{q 0}$, is compatible with the abundance of DE in the universe [1] and (ii) $\rho_{q}$ has already reached the tracking behavior. In other words we have to demand [20]

$$
\text { (a) } \Omega_{q 0}=\bar{\rho}_{q 0}=0.74 \text { and (b) } d^{2} V(\tau=0) / d q^{2} \simeq H_{0}^{2} \text {, }
$$

where we restrict ourselves to the central experimental value of $\Omega_{q 0}$, since, this choice does not affect crucially our results on the CDM abundance.

- Acceleration Constraint. Any successful quintessential model has to account for the presentday acceleration of the universe, i.e., [1] (see also Ref. [43])

$$
-1.12 \leq w_{q}(0) \leq-0.86 \text { (95\% c.l.) with } w_{q}=\left(\dot{q}^{2} / 2-V\right) /\left(\dot{q}^{2} / 2+V\right) .
$$




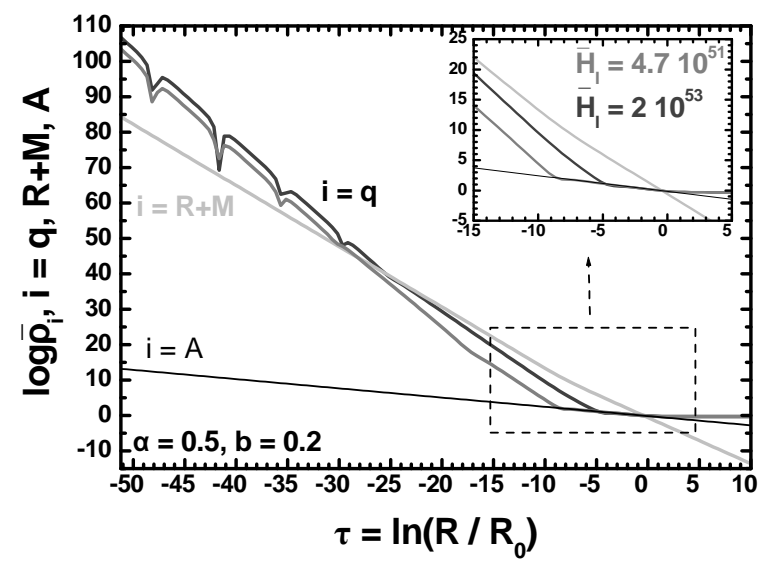

(a)

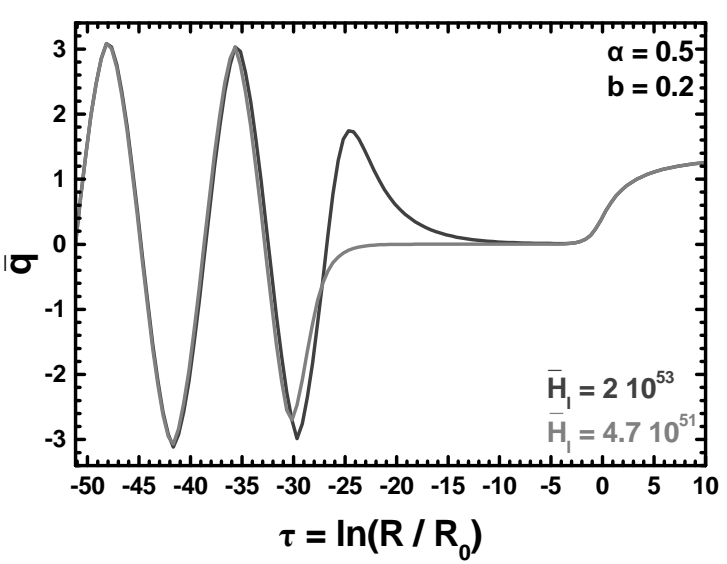

(b)

Figure 2: The evolution of (a) $\log \bar{\rho}_{i}$ with $i=q$ (gray [dark gray] line), $R+M$ (light gray line) and $A$ (thin line); and (b) $\bar{q}$ (gray [dark gray] line) as a function of $\tau=\ln \left(R / R_{0}\right)$ for $\bar{q}_{\mathrm{I}}=0.01, a=0.5, b=0.2, T_{\mathrm{I}}=10^{9} \mathrm{GeV}\left(\tau_{\mathrm{I}} \simeq-51.16\right)$ $M=4.8 \mathrm{eV}$ and $\bar{H}_{\mathrm{I}}=4.7 \cdot 10^{51}\left(\Omega_{q}^{\mathrm{BBN}}=0.0001\right)\left[\bar{H}_{\mathrm{I}}=2 \cdot 10^{53}\left(\Omega_{q}^{\mathrm{BBN}}=0.21\right)\right]$.

2.3.3 Quintessential Dynamics. The cosmological evolution of the various quantities involved in our QKS as a function of $\tau$ is illustrated in Fig. 2 for $\bar{q}_{\mathrm{I}}=0.01, a=0.5, b=0.2, T_{\mathrm{I}}=10^{9} \mathrm{GeV}$ $\left(\tau_{\mathrm{I}} \simeq-51.2\right) M=4.8 \mathrm{eV}$ and $\bar{H}_{\mathrm{I}}=2 \cdot 10^{53}\left(\Omega_{q}^{\mathrm{BBN}}=0.21\right.$ dark gray lines $)$ or $\bar{H}_{\mathrm{I}}=2 \cdot 10^{53}$ $\left(\Omega_{q}^{\mathrm{BBN}}=0.0001\right.$, gray lines). Particularly, in Fig. 2-(a) [Fig. 2-(b)] we draw $\log \bar{\rho}_{i}[\bar{q}]$ versus $\tau$. For $i=q$ (gray and dark gray lines), $\bar{\rho}_{q}$ is computed by inserting in the last equation of Eq. (2.27) the numerical solution of Eq. (2.23). For $i=\mathrm{R}+\mathrm{M}$ (light gray line), we show $\bar{\rho}_{\mathrm{R}+\mathrm{M}}=\bar{\rho}_{\mathrm{R}}+\bar{\rho}_{\mathrm{M}}$ given by Eq. (2.2). For $i=\mathrm{A}$ (thin black line), $\bar{\rho}_{\mathrm{A}}$ is the dimensionless energy density of the attractor solution (see below).

From Fig. 2 we can conclude that $q$ undergoes four phases during its cosmological evolution [20, 21, 44]:

- The kinetic-energy dominated phase during which the evolution of both the universe and $q$ is dominated by $\dot{q} / 2 \gg V$. Therefore $\bar{H} \simeq \bar{H} \bar{q}^{\prime} / \sqrt{2-b \bar{q}^{2}}$ and integrating it we obtain [21]

$$
\bar{q} \simeq \sqrt{\frac{2}{b}} \sin \sqrt{b}\left(\tau-\tau_{\mathrm{I}}\right)
$$

Obviously, for $b>0, q$ is set in harmonic oscillations during the KD era. In particular, $\bar{q}$ develops extrema at

$$
\tau_{\mathrm{ext}} \simeq(2 k+1) \sqrt{\frac{1}{b}} \frac{\pi}{2}+\tau_{\mathrm{I}} \text { with } k=0,1,2, \ldots
$$

On the other hand, $\dot{\bar{q}}=\bar{H} \bar{q}^{\prime}$ almost vanish for $\tau=\tau_{\text {ext }}$. Therefore, at $\tau \simeq \tau_{\text {ext }}, \bar{\rho}_{\mathrm{R}}$ dominates instantaneously over $\dot{q} / 2$. As a consequence, the $\bar{q}$ oscillations become anharmonic. This phase terminates for $\tau=\tau_{\mathrm{KR}}$ where $\rho_{q}=\rho_{\mathrm{R}}$. For the inputs of Fig. 2 we get $\tau_{\mathrm{KR}}=-25.6$ $\left[\tau_{\mathrm{KR}}=-28.2\right]\left(\right.$ or $\left.T_{\mathrm{KR}}=0.02 \mathrm{GeV}\left[T_{\mathrm{KR}}=0.21 \mathrm{GeV}\right]\right)$ for $\bar{H}_{\mathrm{I}}=2 \cdot 10^{53}\left[\bar{H}_{\mathrm{I}}=4.7 \cdot 10^{51}\right] . \mathrm{We}$ observe that the lowest $T_{\mathrm{KR}}$ corresponds to the largest $\bar{H}_{\mathrm{I}}$ (and $\Omega_{q}^{\mathrm{BBN}}$ ). From Fig. 2-(b) we also remark that the height of the fifth peak of $\bar{q}$ decreases with $\bar{H}_{\mathrm{I}}$. In fact, for $\bar{H}_{\mathrm{I}}<4.7 \cdot 10^{51}$ we take $\bar{q}_{0}<0$ and so, $q$ can not serve as quintessence (see below). 


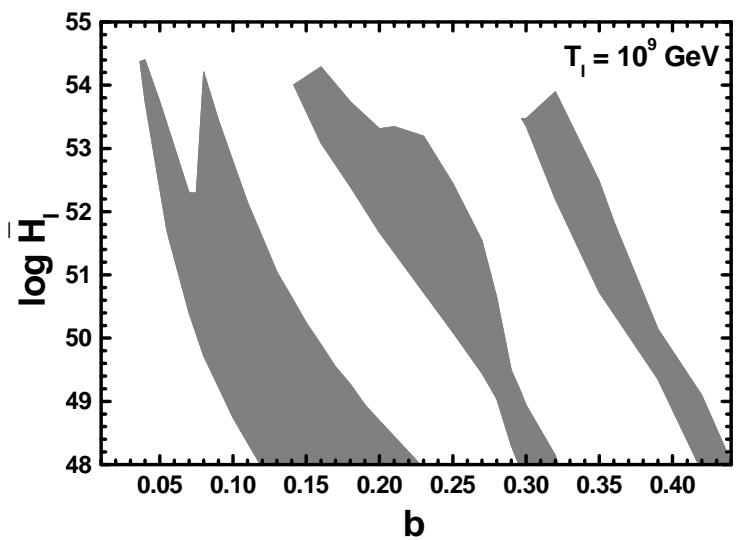

(a)

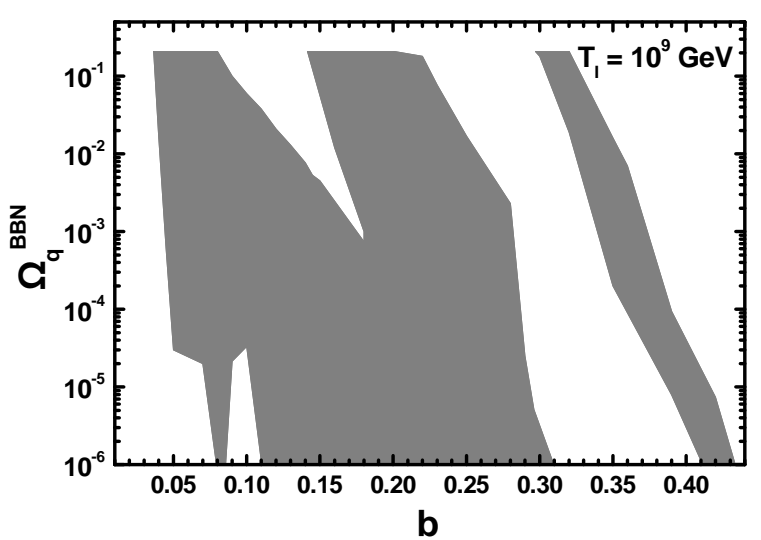

(b)

Figure 3: Allowed (gray shaded) region by Eqs. (2.26) - (2.30) in the (a) $b-\bar{H}_{\mathrm{I}}$ and (b) $b-\Omega_{q}^{\mathrm{BBN}}$ plane for $a=0.5$, $\bar{q}_{\mathrm{I}}=$ 0.01 and $T_{\mathrm{I}}=10^{9} \mathrm{GeV}$.

- The frozen-field dominated phase, where the universe becomes RD and $\rho_{q}$ is dominated initially by $\dot{q} / 2$ and subsequently by $V$ and $\bar{q}$ is stabilized to a constant value - see Fig. 2-(b).

- The attractor dominated phase, where $\rho_{q} \simeq V$ and $\rho_{\mathrm{M}}$ dominates the evolution of the universe. The system in Eq. (2.20) admits [44] a tracking solution since the energy density of the attractor:

$$
\bar{\rho}_{\mathrm{A}} \simeq \bar{\rho}_{\mathrm{Af}} \exp \left(-3\left(1+w_{q}^{\mathrm{fp}}\right)\left(\tau-\tau_{\mathrm{Af}}\right)\right) \text { with } w_{q}^{\mathrm{fp}}=-\frac{2}{a+2}
$$

tracks $\bar{\rho}_{\mathrm{M}}$ until $\tau=\tau_{\mathrm{Af}}$ where the tracking regime terminates and $\bar{\rho}_{\mathrm{M}} \simeq \bar{\rho}_{\mathrm{A}}$. For both $\bar{H}_{\mathrm{I}}$ 's used in Fig. 2, we get $\tau_{\mathrm{Af}}=-0.4$ whereas the onset of this phase occurs at $\tau_{\mathrm{Ai}}=-4.8\left[\tau_{\mathrm{Ai}}=-8.6\right]$ for $\bar{H}_{\mathrm{I}}=2 \cdot 10^{53}\left[\bar{H}_{\mathrm{I}}=4.7 \cdot 10^{51}\right]$. We observe that although the used $\bar{H}_{\mathrm{I}}$ 's differ by two orders of magnitude, both $\bar{\rho}_{q}$ 's reach $\bar{\rho}_{\mathrm{A}}$ highlighting thereby the insensitivity of our QKS to the initial conditions.

- Vacuum Dominated Phase. For $\tau>\tau_{\mathrm{Af}}$, the evolution of the universe is dominated by $V$. For the parameters used in Fig. 2 we get $w_{q}(0) \simeq-0.88$ and $\Omega_{q}(0) \simeq 0.74$.

2.3.4 Allowed Parameter Space. The free parameters of our QKS listed in Table 1 can be restricted using the criteria presented in Sec. 2.3.2. Agreement with Eq. (2.30) entails $0<a \lesssim 0.6$ (compare also with Ref. [45], where less restrictive upper bound on $w_{q}(0)$ is imposed). The parameter $M$ can be determined for every $a$ through Eq. (2.25) so that Eq. (2.29a) is satisfied. The determination of $a$ and $M$ is independent of $\tau_{\mathrm{I}}, \bar{q}_{\mathrm{I}}$ and $\bar{H}_{\mathrm{I}}$ provided that the tracking solution is reached in time. To reduce somehow the parameter space of our investigation we fix $T_{\mathrm{I}}=10^{9} \mathrm{GeV}$ ( or $\tau_{\mathrm{I}}=-51.16$ ). This choice is motivated by the majority of the inflationary models (see, e.g., Ref. [46]). We thereby focus on the two residual free parameters of our model and we design in Fig. 3-(a) [3-(b)] the allowed areas in the $b-\log \bar{H}_{\mathrm{I}}\left[b-\log \Omega_{q}^{\mathrm{BBN}}\right]$ plane. In the gray shaded areas Eqs. (2.26) - (2.30) are fulfilled. Obviously our model possesses an allowed parameter space with a band structure. The upper boundary curves of the allowed bands come from Eq. (2.28). Note, however, that saturation of Eq. (2.28) is not possible for $0.08<b<0.16$.

For any $\left(b, \log \bar{H}_{\mathrm{I}}\right)$, which is consistent with Eq. (2.28) and belongs in a white [gray] band, the resulting $\bar{q}$ after the oscillatory phase turns out to be negative [positive] and so, it cannot [can] serve 
as quintessence. E.g., let us fix $b=0.2$. For $51.7 \lesssim \log \bar{H}_{\mathrm{I}} \lesssim 53.3, \bar{q}$ develops five extrema during its evolution - which is of the type shown in - resulting to $\bar{q}_{0}>0$. Actually in Fig. 2-(b) we display the evolution of $\bar{q}$ as a function of $\tau$ for the two bounds of this band. As $\log \bar{H}_{\text {I }}$ decreases below 53.3 (where the bound of Eq. (2.28) is saturated), the amplitude of the fifth peak, which appears in the $\bar{q}$-evolution (at about $\tau \simeq-24.5$ ) eventually decreases and finally this peak disappears at $\log \bar{H}_{\mathrm{I}} \simeq 51.7$ where the first allowed band terminates. For $48.7 \lesssim \log \bar{H}_{\mathrm{I}} \lesssim 51.7, \bar{q}$ develops four extremes during its evolution resulting to $\bar{q}_{0}<0$. As $\log \bar{H}_{\mathrm{I}}$ decreases below 51.7 the amplitude of the forth peak which appears in the $\bar{q}$-evolution (at about $\tau \simeq-30$ ) decreases and finally this peak disappears at $\log \bar{H}_{\mathrm{I}} \simeq 48.7$ where the second allowed band commences. Note that, in the first allowed band, $\Omega_{q}^{\mathrm{BBN}}$ increases with $\bar{H}_{\mathrm{I}}$ but this is not a generic rule.

Variation of $T_{\mathrm{I}}$ (or equivalently $\tau_{\mathrm{I}}$ ) modifies the range of the obtainable $\Omega_{q}^{\mathrm{BBN}}$ and changes somehow the position of the several bands of our parameter space but do not alter the essential features of our results. E.g., for $b=0.2$ and $T_{\mathrm{I}}=10^{10} \mathrm{GeV}\left[T_{\mathrm{I}}=10^{8} \mathrm{GeV}\right]$ (or $\tau_{\mathrm{I}}=-53.5$ $\left.\left[\tau_{\mathrm{I}}=-48.9\right]\right)$ the margin of the first allowed band is $53.6 \lesssim \log \bar{H}_{\mathrm{I}} \lesssim 56.4$ [49.6 $\left.\lesssim \log \bar{H}_{\mathrm{I}} \lesssim 51.1\right]$ and the second allowed band commences at $\log \bar{H}_{\mathrm{I}} \simeq 50.7\left[\log \bar{H}_{\mathrm{I}} \simeq 46.75\right]$.

\section{The WIMP Relic Density}

We turn to the calculation of the relic density, $\Omega_{\chi} h^{2}$, of a WIMP-CDM candidate, $\chi$. Employing the symbols defined in Eq. (2.9), $\Omega_{\chi} h^{2}$ can be found from the well-know formula:

$$
\Omega_{\chi}=\frac{\rho_{\chi 0}}{\rho_{\mathrm{c} 0}}=\left.\frac{s_{0}}{\rho_{\mathrm{c} 0}} \frac{m_{\chi} n_{\chi}}{s}\right|_{\tau_{\mathrm{f}}} \Rightarrow \Omega_{\chi} h^{2}=\left.4.533 \cdot 10^{-27} \mathrm{GeV}^{2} \frac{m_{\chi} f_{\chi}}{s R^{3}}\right|_{\tau_{\mathrm{f}}},
$$

where $s_{0} h^{2} / \rho_{\mathrm{c} 0}^{1 / 4}=4.533 \cdot 10^{-27} \mathrm{GeV}^{2}$ and $\tau_{\mathrm{f}}$ is a value of $\tilde{\tau}[\tau]$ for the LRS [QKS] - see Eq. (2.10) [Eq. (2.21)] - large enough so as $f_{\chi}$ is stabilized to its present constant value, $f_{\chi 0}$. Recall that $s R^{3}$ in the denominator of Eq. (3.1) remains constant only for $\tilde{\tau}>\tilde{\tau}_{R H}$ in the LRS but for every $\tau$ in the QKS - see Table 1. The evolution of $f_{\chi}$ obeys a Boltzmann equation. In Sec. 3.1 we present and solve this Boltzmann equation and in Sec. 3.2 we investigate how we can achieve within our scenaria an enhancement of $\Omega_{\chi} h^{2}$ w.r.t its value in the SC.

\subsection{The Boltzmann Equation}

Since $\chi$ 's are in kinetic equilibrium with the cosmic fluid, their number density, $n_{\chi}$, evolves according to the Boltzmann equation:

$$
\dot{n}_{\chi}+3 H n_{\chi}+\langle\sigma v\rangle\left(n_{\chi}^{2}-n_{\chi}^{\mathrm{eq} 2}\right)= \begin{cases}\Gamma_{\phi} N_{\chi} n_{\phi} & \text { for the LRS } \\ 0 & \text { for the QKS }\end{cases}
$$

where $H$ is found from Eq. (2.1). In order to find a precise numerical solution to our problem, we have to solve Eq. (3.2) together with Eq. (2.20) [Eqs. (2.6a) and (2.6b)] for the QKS [LRS]. To this end, we rewrite Eq. (3.2) in terms of the quantities defined in Eq. (2.9) as follows

$$
\bar{H} R^{3} f_{\chi}^{\prime}+\overline{\langle\sigma v\rangle}\left(f_{\chi}^{2}-f_{\chi}^{\mathrm{eq} 2}\right)= \begin{cases}\bar{\Gamma}_{\phi} N_{\chi} \bar{n}_{\phi} R^{6} & \text { for the LRS, } \\ 0 & \text { for the QKS, }\end{cases}
$$

where $\bar{H}$ given by Eq. (2.12a) [Eq. (2.24)] and prime in this section denotes derivation w.r.t $\tau=\tilde{\tau}$ $[\tau=\tau]$ for the LRS [QKS]. Eq. (3.3) can be solved numerically in conjunction with Eq. (2.11) 
[Eq. (2.23)] for the LRS [QKS]. In the LRS, we use the initial conditions in Eq. (2.13) and we integrate from $\tilde{\tau}=0$ to $\tilde{\tau}_{\mathrm{f}} \simeq 50$ (with $g$ 's fixed to their values at $T_{\mathrm{RH}}$ ). In the QKS, we impose the initial condition $\bar{n}_{\chi}\left(\tau_{\chi}\right)=\bar{n}_{\chi}^{\mathrm{eq}}\left(\tau_{\chi}\right)$, where $\tau_{\chi}$ corresponds to the beginning $(x=1)$ of the Boltzmann suppression of $\bar{n}_{\chi}^{\mathrm{eq}}$ in Eq. (2.7). The integration of Eq. (3.3) is realized from $\tau_{\mathrm{I}}$ down to $\tau_{\mathrm{BBN}} \simeq$ -22.5 (an integration until to 0 gives also the same result).

Based on the semi-analytical expressions of Sec. 2, we can proceed to an approximate computation, which facilitates the understanding of the problem and gives, in most cases, accurate results. In particular, we can set - see Eq. (2.24) [Eqs. (2.17) and (2.18)] for the QKS [LRS]:

$$
\begin{aligned}
& \bar{H} \simeq \sqrt{\bar{\rho}_{\mathrm{R}} g_{\mathrm{C}}} \text { where } g_{\mathrm{C}} \simeq\left\{\begin{array}{ll}
1+T^{4} / T_{\mathrm{RH}}^{4} & \text { for } \tilde{\tau} \ll \tilde{\tau}_{\mathrm{RH}}, \\
1 & \text { for } \tilde{\tau} \gg \tilde{\tau}_{\mathrm{RH}}
\end{array}\right. \text { for the LRS, or } \\
& g_{\mathrm{C}} \simeq \frac{1}{\left(1-b \bar{q}^{2} / 2\right)}\left\{\begin{array}{ll}
1+f_{q}^{2} / 2 R^{6} \bar{\rho}_{\mathrm{R}} & \text { for } \tau \ll \tau_{\mathrm{KR}}, \\
1 & \text { for } \tau \gg \tau_{\mathrm{KR}}
\end{array}\right. \text { for the QKS. }
\end{aligned}
$$

Introducing the notion of freeze-out temperature, $T_{\mathrm{F}}=T\left(\tau_{\mathrm{F}}\right)=x_{\mathrm{F}} m_{\chi}$ (see, e.g., Ref. [11, 16] and references therein) we are able to study Eq. (3.3) in the two extreme regimes:

- For $\tau \ll \tau_{\mathrm{F}}, f_{\chi} \simeq f_{\chi}^{\mathrm{eq}}$. So, it is more convenient to rewrite Eq. (3.3) in terms of the variable $\Delta(\tau)=f_{\chi}(\tau)-f_{\chi}^{\mathrm{eq}}(\tau)$ as follows:

$$
\bar{H} R^{3}\left(\Delta^{\prime}+f_{\chi}^{\mathrm{eq}}\right)+\overline{\langle\sigma v\rangle} \Delta\left(\Delta+2 f_{\chi}^{\mathrm{eq}}\right)= \begin{cases}\bar{\Gamma}_{\phi} N_{\chi} \bar{n}_{\phi} R^{6} & \text { for the LRS } \\ 0 & \text { for the QKS. }\end{cases}
$$

The freeze-out point $\tau_{\mathrm{F}}$ can be defined by $\Delta\left(\tau_{\mathrm{F}}\right)=\delta_{\mathrm{F}} f_{\chi}^{\mathrm{eq}}\left(\tau_{\mathrm{F}}\right)$ where $\delta_{\mathrm{F}}$ is a constant of order unity, determined by comparing the exact numerical solution of Eq. (3.3) with the approximate under consideration one. Inserting this definition into Eq. (3.5), we obtain the equation:

$$
\begin{aligned}
& \left(\delta_{\mathrm{F}}+1\right) f_{\chi}^{\mathrm{eq}}\left(\tau_{\mathrm{F}}\right) \bar{H} R^{3}\left(\ln f_{\chi}^{\mathrm{eq}}\right)^{\prime}\left(\tau_{\mathrm{F}}\right)+\delta_{\mathrm{F}}\left(\delta_{\mathrm{F}}+2\right) \overline{\langle\sigma v\rangle} f_{\chi}^{\mathrm{eq} 2}\left(\tau_{\mathrm{F}}\right)= \begin{cases}\bar{\Gamma}_{\phi} N_{\chi} \bar{n}_{\phi} R^{6} & \begin{array}{l}
\text { for the LRS, } \\
0
\end{array} \\
\text { with }\left(\ln f_{\chi}^{\mathrm{eq}}\right)^{\prime}(\tau)=3+x^{\prime} \frac{(16+3 x)(18+25 x)}{2 x^{2}(8+15 x)}\end{cases}
\end{aligned}
$$

which can be solved w.r.t $\tau_{\mathrm{F}}$ iteratively. The $\tau-x[\tilde{\tau}-x]$ dependence can be derived by combining Eq. (2.7) and Eq. (2.2) [Eq. (2.12)] for the QKS [LRS].

- For $\tau \gg \tau_{\mathrm{F}}, f_{\chi} \gg f_{\chi}^{\mathrm{eq}}$ and so, we can set $f_{\chi}^{2}-f_{\chi}^{\mathrm{eq} 2} \simeq f_{\chi}^{2}$ in Eq. (3.3). Let us analyze this case for each scenario separately:

- In the LRS and for the range of the parameters under consideration - see Sec. 3.2 - we single out two cases:

* Dominant non-thermal production (non-TP). In this case, which is mainly applicable for very low $T_{\mathrm{RH}}$ 's, $f_{\chi}^{2} \overline{\langle\sigma v\rangle} \ll \bar{\Gamma}_{\phi} N_{\chi} \bar{n}_{\phi} R^{6}$. Therefore, Eq. (3.3) can be integrated analytically inserting into it Eq. (2.17a) as follows:

$$
f_{\chi 0} \simeq \frac{2}{3} \sqrt{\bar{\rho}_{\phi_{\mathrm{I}}}} \bar{\Gamma}_{\phi} N_{\chi} \Delta_{\phi}^{-1} \bar{m}_{\phi}^{-1}\left(e^{3 \tilde{\tau}_{\mathrm{RH}} / 2}-e^{3 \tilde{\mathrm{\tau}}_{\mathrm{F}} / 2}\right) .
$$

Since $f_{\chi 0}$ takes its main contribution close to $\tilde{\tau}_{R H} \gg \tilde{\tau}_{F}$ our result is more or less independent of $\tilde{\tau}_{\mathrm{F}}$. 
* Equipartition between TP and non-TP. Besides $f_{\chi}^{\text {eq }}$ none of the other terms of Eq. (3.3) can be neglected in this case. A rather precise result for $f_{\chi 0}$ can be obtained by numerically integrating Eq. (3.3) subsequently from (i) $\tilde{\tau}=\tilde{\tau}_{F}$ until $\tilde{\tau}=\tilde{\tau}_{R H}$ with initial condition $f_{\chi}\left(\tilde{\tau}_{\mathrm{F}}\right)=\left(\delta_{\mathrm{F}}+1\right) f_{\chi}^{\mathrm{eq}}\left(\tilde{\tau}_{\mathrm{F}}\right)$ and using $\bar{H}\left[\bar{\rho}_{\phi}\right]$ given by Eq. (3.4) [Eq. (2.17a)]; (ii) $\tilde{\tau}=\tilde{\tau}_{\mathrm{RH}}$ until $\tilde{\tau}=\tilde{\tau}_{\mathrm{f}} \simeq 50$, employing the estimates of Eq. (2.19) for $\bar{\rho}_{\phi}$ and $\bar{\rho}_{\mathrm{R}}$. A less accurate result for this case can be derived $[9,10]$ by equating the annihilation rate $\Gamma_{\chi}=n_{\chi}\langle\sigma v\rangle$ to the expansion rate $H$ at the completion of reheating. Combining Eq. (2.17a) and Eq. (2.18) we obtain $\bar{H}\left(\tilde{\tau}_{\mathrm{RH}}\right)=2 \bar{\rho}_{\phi}\left(\tilde{\tau}_{\mathrm{RH}}\right)=2 \sqrt{2} \bar{\Gamma}_{\phi} / 5$ and so, we arrive at:

$$
\frac{n_{\chi}}{s} \simeq \frac{9 \sqrt{2}}{\pi^{2}} \frac{\Gamma_{\phi}}{\langle\sigma v\rangle T_{\mathrm{RH}}^{3}}
$$

In general, this result underestimates the numerical one by a factor of unity. However, the method applied reveals the presence of the phenomenon of reannihilation [47] in this case, i.e., the occurrence of a secondary (for $\tilde{\tau} \gg \tilde{\tau}_{\mathrm{F}}$ ) $\chi$ decoupling - see Sec. 3.2.1.

- In the QKS, we can integrate numerically Eq. (3.3) from $\tau_{\mathrm{F}}$ down to 0, as follows:

$$
f_{\chi 0}=\left(f_{\chi \mathrm{F}}^{-1}+J_{\mathrm{F}}\right)^{-1}, \text { where } J_{\mathrm{F}}=\int_{\tau_{\mathrm{F}}}^{0} d \tau \frac{\overline{\langle\sigma v\rangle}}{\bar{H} R^{3}} \text { and } f_{\chi \mathrm{F}}=\left(\delta_{\mathrm{F}}+1\right) f_{\chi}^{\mathrm{eq}}\left(\tau_{\mathrm{F}}\right) .
$$

Although not crucial, a choice $\delta_{\mathrm{F}}=1.2 \mp 0.2$ assists us to approach better the precise numerical solution of Eq. (3.3).

\subsection{The Enhancement of $\Omega_{\chi} h^{2}$}

As we explain in Sec. 4 the interpretation of the $e^{ \pm}$-CR anomalies favors $10^{-7} \lesssim\langle\sigma v\rangle / \mathrm{GeV}^{-2} \lesssim$ $10^{-6}$ which results to $\left.0.0025 \gtrsim \Omega_{\chi} h^{2}\right|_{\mathrm{SC}} \gtrsim 0.00027$ for $0.1 \leq m_{\chi} / \mathrm{TeV} \leq 3$, where $\left.\Omega_{\chi} h^{2}\right|_{\text {SC }}$ denotes $\Omega_{\chi} h^{2}$ within the SC. Clearly, the resulting $\left.\Omega_{\chi} h^{2}\right|_{\text {SC }}$ lie much lower than the range of Eq. (1.2). However, the proposed non-standard scenaria can increase $\Omega_{\chi} h^{2}$ w.r.t $\left.\Omega_{\chi} h^{2}\right|_{\text {SC }}$. The resulting enhancement can be quantified, by defining the quantity:

$$
\Delta \Omega_{\chi}=\left(\Omega_{\chi} h^{2}-\left.\Omega_{\chi} h^{2}\right|_{\mathrm{SC}}\right) /\left.\Omega_{\chi} h^{2}\right|_{\mathrm{SC}} .
$$

We below analyze the behavior of $\Delta \Omega_{\chi}$ as a function of the free parameters of each non-standard scenario separately.

3.2.1 THE LRS. Let us initially clarify that in the LRS, both signs of $\Delta \Omega_{\chi}$ are possible, as emphasized in Ref. [11, 12]. However, we here confine ourselves to the combination of parameters which assures the favored from the $e^{ \pm}$-CR data $\Delta \Omega_{\chi}>0$. The dependence of $\Delta \Omega_{\chi}$ on the free parameters of the LRS can be inferred from Fig. 4, where we depict $\Delta \Omega_{\chi}$ versus $T_{\mathrm{RH}}$ for $m_{\chi}=0.5 \mathrm{TeV}$, $\langle\sigma v\rangle=10^{-6} \mathrm{GeV}^{-2}\left[\langle\sigma v\rangle=10^{-7} \mathrm{GeV}^{-2}\right]$ (gray [light gray] lines) and $c_{\chi \phi}=1$ (solid lines), $c_{\chi \phi}=10^{-4}$ (dashed lines) and $c_{\chi \phi}=10^{-6}$ (dotted lines). The ranges of parameters where each production mechanism is activated are also shown in the table included. Note that the exposed ranges depend very weakly on the employed $m_{\chi}$ 's and $\langle\sigma v\rangle$ 's. We observe that $\Delta \Omega_{\chi}$ increases with $T_{\mathrm{RH}}$ when we have non-TP as expected from Eq. (3.7), but it decreases as $T_{\mathrm{RH}}$ increases when we have equipartition between non-TP and TP, as anticipated in Eq. (3.8). The former mechanism is dominant mainly for very low $T_{\mathrm{RH}}$ 's whereas the latter is present for higher $T_{\mathrm{RH}}$ 's. The accuracy 


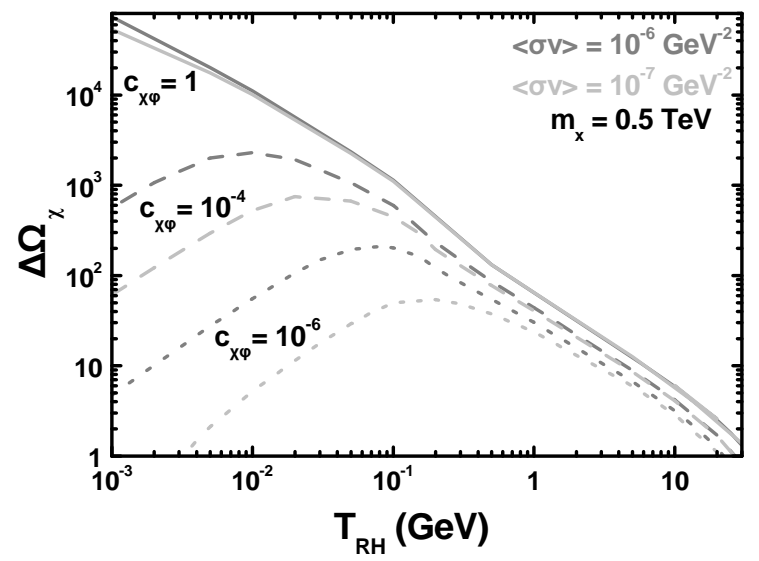

\begin{tabular}{|c|c|c|}
\hline$c_{\chi \phi}$ & $T_{\mathrm{RH}}(\mathrm{GeV})$ & $\chi$ Production \\
\hline \hline $10^{-6}$ & $0.001-0.2$ & NON-TP \\
& $0.2-30$ & NON-TP + TP \\
\hline $10^{-4}$ & $0.001-0.02$ & NON-TP \\
& $0.02-30$ & NON-TP + TP \\
\hline 1 & $0.001-30$ & NON-TP+TP \\
\hline
\end{tabular}

FiguRE 4: $\Delta \Omega_{\chi}$ versus $T_{\mathrm{RH}}$ for the LRS with $m_{\chi}=0.5 \mathrm{TeV},\langle\sigma v\rangle=10^{-6} \mathrm{GeV}^{-2}\left[\langle\sigma v\rangle=10^{-7} \mathrm{GeV}^{-2}\right]$ (gray [light gray] lines) and $c_{\chi \phi}=1$ (solid lines), $c_{\chi \phi}=10^{-4}$ (dashed lines) and $c_{\chi \phi}=10^{-6}$ (dotted lines). In the table we also show the type of the $\chi$ production for each $c_{\chi \phi}$ and the various ranges of $T_{\mathrm{RH}}$.

of the corresponding empirical expressions in Eq. (3.7) [Eq. (3.8)] increases as $T_{\mathrm{RH}}$ decreases [increases] and as $\langle\sigma v\rangle$ decreases [increases]. It is remarkable that for $c_{\chi \phi}$ 's where both production mechanisms are possible (e.g., $c_{\chi \phi}=10^{-6}$ or $10^{-4}$ ) we can obtain the same $\Delta \Omega_{\chi}$ for two values of $T_{\mathrm{RH}}$. In general, $\Delta \Omega_{\chi}$ increases with $N_{\chi}$. Augmentation of $m_{\chi}$ increases $\Omega_{\chi} h^{2}$, too, but does not alter the dependence of $\Omega_{\chi} h^{2}$ on $T_{\mathrm{RH}}$ and the ranges where the $\chi$-production mechanisms are activated.

The operation of the two types of $\chi$ production encountered in Fig. 4 is visualized in Fig. 5(a) and (b). In these, we display the actual $\chi$ yield, $n_{\chi} / s$ (solid lines) and its equilibrium value, $n_{\chi}^{\mathrm{eq}} / s$ (dashed lines) the dimensionless actual interaction rate of $\chi, \bar{\Gamma}_{\chi}=\bar{n}_{\chi} \overline{\langle\sigma v\rangle}$ (solid lines), its equilibrium value, $\bar{\Gamma}_{\chi}^{\mathrm{eq}}=\bar{n}_{\chi}^{\mathrm{eq}} \overline{\langle\sigma v\rangle}$ (dotted lines) and the dimensionless expansion rate $\bar{H}$ (dashed lines) - given by Eq. (2.12a) - versus $\tilde{\tau}$. In both figures we use $m_{\chi}=0.5 \mathrm{TeV}, m_{\phi}=100 \mathrm{TeV}$, $\langle\sigma v\rangle=3 \cdot 10^{-7} \mathrm{GeV}^{-2}$ and $\left(N_{\chi}, T_{\mathrm{RH}}\right)=(1,0.5 \mathrm{GeV})\left[\left(N_{\chi}, T_{\mathrm{RH}}\right)=\left(7.5 \cdot 10^{-5}, 1 \mathrm{MeV}\right)\right]$ (gray [light gray] lines). For the selected parameters, the evolution of the background energy densities $\left(\log \bar{\rho}_{\phi}\right.$ and $\left.\log \bar{\rho}_{\mathrm{R}}\right)$ and $T$ is presented in Fig. 1-(a) and (b). The completion of reheating occurs at $\tilde{\tau}_{\mathrm{RH}} \simeq 36.1\left[\tilde{\tau}_{\mathrm{RH}}=45.1\right]$ for $T_{\mathrm{RH}}=0.5 \mathrm{GeV}\left[T_{\mathrm{RH}}=1 \mathrm{MeV}\right]$.

From Fig. 5-(a) we can deduce that $n_{\chi} / s$ takes its present value close to [clearly above] $\tilde{\tau}_{R H}$ for $T_{\mathrm{RH}}=1 \mathrm{MeV}\left[T_{\mathrm{RH}}=0.5 \mathrm{GeV}\right]$. For this reason, the integration of Eq. (3.3) until $\tilde{\tau}_{\mathrm{RH}}$ for non-TP is sufficient for an accurate result - see Eq. (3.7) -, but insufficient when non-TP and TP interplay. The $\chi$ reannihilation takes place along the almost vertical part of the gray line for $\tilde{\tau}$ a little lower than $\tilde{\tau}_{\mathrm{RH}}$. It is notable that in Ref. $[11,12]$ which focus on lower $\langle\sigma v\rangle$ 's than the ones considered here, the phenomenon of reannihilation is not stressed.

This effect is further analyzed, following the approach of the first paper in Ref. [47], in Fig. 5(b). From this we infer that for $T_{\mathrm{RH}}=1 \mathrm{MeV}$, where non-TP outstrips, $\chi$ decouples from plasma only once at $\tilde{\tau}_{\mathrm{F}}=16.5$ where $\Gamma_{\chi}=\Gamma_{\chi}^{\mathrm{eq}}=H$ and an intersection of the light gray lines is observed (note that the light gray and gray dashed lines coincide at that region). On the contrary, for $T_{\mathrm{RH}}=$ $0.5 \mathrm{GeV}$, where non-TP and TP coexist, we observe that $\chi$ decouples from plasma initially at about $\tilde{\tau}_{\mathrm{F}}=26.3$ where $\Gamma_{\chi}^{\mathrm{eq}}=H$ but also at $\tilde{\mathrm{\tau}}_{\mathrm{Fr}}=37.9>\tilde{\tau}_{\mathrm{RH}}$ where $\Gamma_{\chi}=H$. In other words, we observe two intersections between the two pairs of the three gray lines. This effect signalizes the existence 


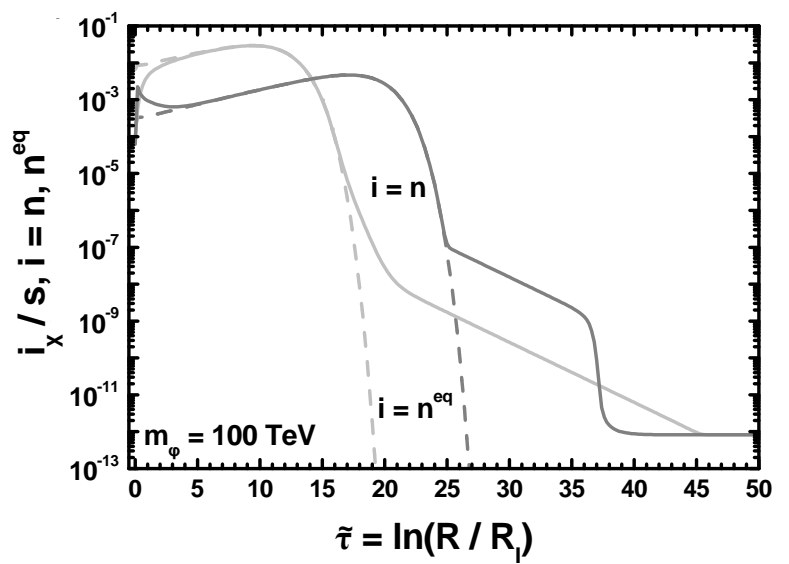

(a)

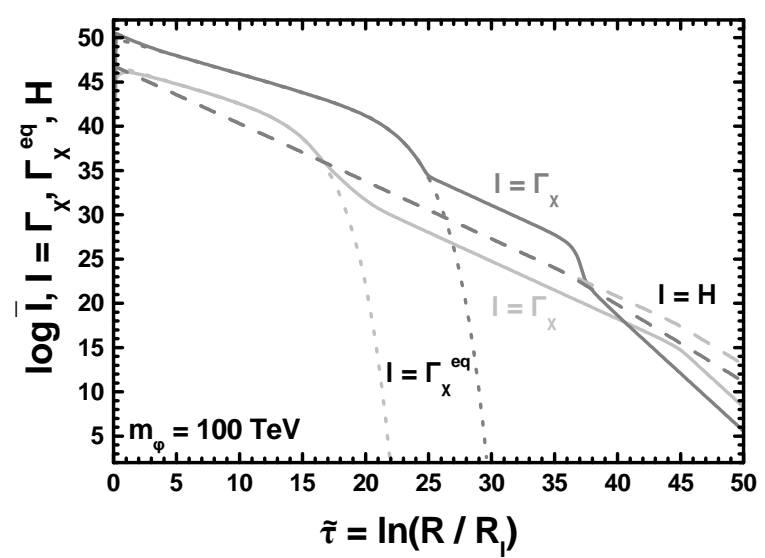

(b)

$$
\begin{aligned}
& \mathrm{T}_{\mathrm{RH}}=1 \quad \mathrm{MeV}, \mathrm{N}_{\mathrm{x}}=7.510^{-5} \\
& \mathrm{~T}_{\mathrm{RH}}=0.5 \mathrm{GeV}, \mathrm{N}_{\mathrm{x}}=1
\end{aligned}
$$

Figure 5: (a) $n_{\chi} / s$ (solid lines) and $n_{\chi}^{\mathrm{eq}} / s$ (dashed lines) versus $\tilde{\mathrm{\tau}}$ and (b) $\bar{\Gamma}_{\chi}$ (solid lines), $\bar{\Gamma}_{\chi}^{\mathrm{eq}}$ (dotted lines) and $\bar{H}$ (dashed lines) versus $\tilde{\tau}$ for the LRS with $m_{\chi}=0.5 \mathrm{TeV}, m_{\phi}=100 \mathrm{TeV},\langle\sigma v\rangle=3 \cdot 10^{-7} \mathrm{GeV}^{-2}$ and $\left(N_{\chi}, T_{\mathrm{RH}}\right)=(1,0.5 \mathrm{GeV})$ $\left[\left(N_{\chi}, T_{\mathrm{RH}}\right)=\left(7.5 \cdot 10^{-5}, 1 \mathrm{MeV}\right)\right]$ (gray [light gray] lines) - in both cases, we get $\Omega_{\chi} h^{2}=0.11$.

of a period of $\chi$ reannihilation similar to that noticed in Ref. [47]. Contrary to that situation, in our case (i) $\Gamma_{\chi}$ remains larger than $H$ after the first $\chi$ decoupling and drops sharply below $H$ after reannihilation, and (ii) $H$ smoothly evolves from its behavior during LRS to that within RD era.

3.2.2 THE QKS. The presence of $g_{\mathrm{C}}>1$ in Eq. (3.6) and, mainly, in Eq. (3.9) reduces $J_{\mathrm{F}}$ w.r.t its value in the SC generating, thereby, $\Delta \Omega_{\chi}>0$ within the QKS. The mechanism of the $\chi$ decoupling in this case, for both $b=0$ and $b \neq 0$, is pretty known - see Ref. [8, 14, 16]. However, a peculiar effect emerges in the dependence of $\Delta \Omega_{\chi}$ on $m_{\chi}$ for $b \neq 0$ which can be inferred from Fig. 6, where we display $\Delta \Omega_{\chi}$ versus $m_{\chi}$ for $a=0.5, \bar{H}_{\mathrm{I}}=6.3 \cdot 10^{53},\langle\sigma v\rangle=10^{-6} \mathrm{GeV}^{-2}\left[\langle\sigma v\rangle=10^{-7} \mathrm{GeV}^{-2}\right]$ (gray [light gray] lines) and $b=0$ (solid lines), $b=0.15$ (dashed lines) and $b=0.32$ (dotted lines). The chosen $\bar{H}_{\mathrm{I}}$ 's result to $\Omega_{q}^{\mathrm{BBN}} \simeq 0.01,0.068$ or 0.19 for $b=0,0.15$ or 0.32 correspondingly.

Obviously, for $b=0$ we get a pure KD era and our results reduce to those presented in Ref. [16], i.e., $\Delta \Omega_{\chi}$ increases when $m_{\chi}$ increases or $\langle\sigma v\rangle$ decreases. On the contrary, for $b \neq 0$, $\Delta \Omega_{\chi}$ depends crucially on the hierarchy between $\tau_{\mathrm{F}}$ and $\tau_{\text {ext }}$ found from Eqs. (3.6) and (2.32) respectively. Given that $J_{\mathrm{F}}$ takes its main contribution from $g_{\mathrm{C}}$ for $\tau \sim \tau_{\mathrm{F}}, J_{\mathrm{F}}$ is enhanced - see Eq. (3.9) - if $\tau_{\mathrm{F}}$ is lower than $\tau_{\text {ext }}$ and close to it, since $g_{\mathrm{C}}$ is suppressed $\left(g_{\mathrm{C}} \simeq 1\right)$ for $\tau \simeq \tau_{\text {ext }}$. As a consequence - see Eqs. (3.1) and (3.9) $-\Delta \Omega_{\chi}$ diminishes. This argument is highlighted in the table of Fig. 6. There, we list the range of $\tau_{\mathrm{F}}$ for $0.1 \leq m_{\chi} / \mathrm{TeV} \leq 3$ and $\langle\sigma v\rangle=10^{-7} \mathrm{GeV}^{-2}$ or $\langle\sigma v\rangle=10^{-6} \mathrm{GeV}^{-2}$ and the logarithmic time $\tau_{\text {ext }}$ at which the closest to $\tau_{\mathrm{F}}$ 's peak in the $q$ evolution takes place for $b=0.15$ or $b=0.32$ and $\bar{H}_{\mathrm{I}}=6.3 \cdot 10^{53}$. Clearly $\tau_{\mathrm{F}}\left[\tau_{\text {ext }}\right]$ is independent of $b$ and $\bar{H}_{\mathrm{I}}\left[m_{\chi}\right.$ and $\left.\langle\sigma v\rangle\right]$. As $m_{\chi}$ increases above $0.1 \mathrm{TeV}, \tau_{\mathrm{F}}$ moves closer to $\tau_{\text {ext }}$ and $\Delta \Omega_{\chi}$ decreases with its minimum $\left.\Delta \Omega_{\chi}\right|_{\min }$ occurring at $\tau_{\mathrm{F}}^{\min } \simeq \tau_{\text {ext }}$. The small deviation of $\tau_{\mathrm{F}}^{\min }$ from $\tau_{\text {ext }}$ can be attributed to the presence of $f_{\chi \mathrm{F}}$ in Eq. (3.9). The appearance of the minima can be avoided if $\tau_{\mathrm{F}}$ 's happen to remain constantly lower than $\tau_{\text {ext }}$ 's - see, e.g., Fig. 9- $\left(c_{2}\right)$. Variation of $T_{\mathrm{I}}$ or $\bar{H}_{\mathrm{I}}$ leads to a displacement of $\tau_{\text {ext }}$ 's - see Eq. (2.32) - relocating, thereby, the minima of $\Delta \Omega_{\chi}$ in Fig. 6. However, our conclusions on the behavior of $\Delta \Omega_{\chi}$ remain intact. 


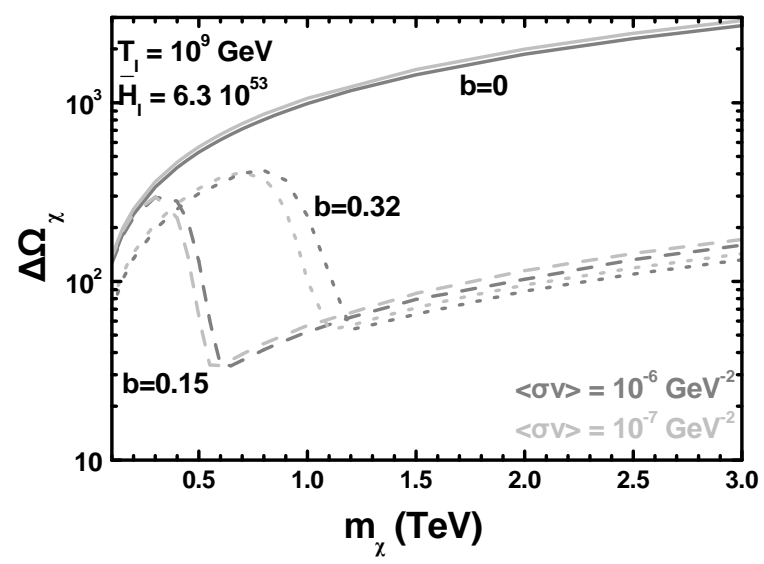

\begin{tabular}{|c|c|c|}
\hline $\begin{array}{c}\langle\sigma v\rangle \\
\left(\mathrm{GeV}^{-2}\right)\end{array}$ & $10^{-7}$ & $10^{-6}$ \\
\hline \hline$-\tau_{\mathrm{F}}$ & $31.7-35.2$ & $31.6-35.1$ \\
\hline \hline \multirow{2}{*}{$\tau_{\mathrm{F}}^{\min }$} & \multicolumn{2}{|c|}{$b=0.15, \tau_{\mathrm{ext}} \simeq-33.1$} \\
\cline { 2 - 3 } & -33.3 & -33.3 \\
\hline \hline \multirow{2}{*}{$\tau_{\mathrm{F}}^{\min }$} & \multicolumn{2}{|c|}{$b=0.32, \tau_{\mathrm{ext}} \simeq-33.8$} \\
\cline { 2 - 3 } & -34.1 & -34.0 \\
\hline
\end{tabular}

Figure 6: $\Delta \Omega_{\chi}$ versus $m_{\chi}$ for the $Q K S$ with $a=0.5, \bar{H}_{\mathrm{I}}=6.3 \cdot 10^{53}, T_{\mathrm{I}}=10^{9} \mathrm{GeV},\langle\sigma v\rangle=10^{-6} \mathrm{GeV}^{-2}[\langle\sigma v\rangle=$ $10^{-7} \mathrm{GeV}^{-2}$ ] (gray [light gray] lines) and $b=0$ (solid lines), $b=0.15$ (dashed lines) and $b=0.32$ (dotted lines). In the table we also show the range of $\tau_{\mathrm{F}}$ for $0.1 \leq m_{\chi} / \mathrm{TeV} \leq 3$, the freeze-out logarithmic time $\tau_{\mathrm{F}}^{\mathrm{min}}$ at which the minima of $\Delta \Omega_{\chi}$ occur and the closest to $\tau_{\mathrm{F}}^{\mathrm{min}}$ 's $\tau_{\mathrm{ext}}$ 's for the selected $b$ 's.

\section{PAMELA, ATIC and Fermi-LAT Anomalies}

The aforementioned $\Delta \Omega_{\chi}$ obtained within the LRS or QKS assists us to explain the experimental data on the $e^{ \pm}$-CRs consistently with Eq. (1.2). Indeed, the observed anomalies on the CR $e^{ \pm}$fluxes can be attributed to the annihilation of $\chi$ 's in the galaxy provided that $m_{\chi}$ and $\langle\sigma v\rangle$ are chosen appropriately. In Sec. 4.1 we outline the basic formalism that we employ in order to estimate the observable quantities as a function of these parameters and in Sec. 4.2 we display our fittings.

\subsection{Cosmic Rays from AnNihilation of WIMPs}

After being produced in the Milky Way halo, charged CRs propagate in the galaxy and its vicinity in a rather complicated way before reaching the earth. Their propagation is commonly evaluated by solving a diffusion equation $[48,49]$ with static cylindrical boundary conditions. The solution can be casted into the following semi-analytical form $[48,49,50]$ which yields the $e^{+}$flux per energy - in units $\mathrm{GeV}^{-1} \mathrm{~cm}^{-2} \mathrm{~s}^{-1} \mathrm{sr}^{-1}-$ at earth from the $\chi$ annihilation:

$$
\Phi_{e^{+}}^{\chi \chi}(E)=\frac{1}{2} \frac{v_{e^{+}}}{4 \pi b(E)}\left(\frac{\rho_{\odot}}{m_{\chi}}\right)^{2}\langle\sigma v\rangle \int_{E}^{m_{\chi}} d E^{\prime} I\left(\lambda_{D}\left(E, E^{\prime}\right)\right) \frac{d N_{e^{+}}}{d E_{e^{+}}^{\prime}},
$$

where $v_{e^{+}}$is the velocity of $e^{+}$which is practically equal to the one of the light, the pre-factor of $1 / 2$ arises from our assumption that $\chi$ is a Majorana particle, $\rho_{\odot}=0.3 \mathrm{GeV} / \mathrm{cm}^{3}$ is the local CDM density, $b(E)=E^{2} /\left(\mathrm{GeV} t_{E}\right)$ with $t_{E}=10^{16} \mathrm{~s}$ is the energy loss rate function and $d N_{e^{+}} / d E_{e^{+}}$denotes the energy distribution of $e^{+}$'s per $\chi$ annihilation. Motivated by the highly restrictive PAMELA data [51] on the anti-proton mode of the CDM annihilation, we consider a purely leptophilic $\chi$ (however, see also Ref. [52]). In particular, we incorporate in our investigation the following annihilation modes: $\chi \chi \rightarrow e^{-} e^{+}, \chi \chi \rightarrow \mu^{-} \mu^{+}$or $\chi \chi \rightarrow \tau^{-} \tau^{+}$. In the first case, $d N_{e^{+}} / d E_{e^{+}}$is given by a simple analytic expression [53] whereas in both latter cases, we use analytic parametrizations (presented in Ref. [54, 55]) of $d N_{e^{+}} / d E_{e^{+}}$which reproduce quite accurately the numerical outputs of the package Pyтнia [56]. In all cases the effect of final state radiation [57] is taken into account. 
Namely, we take

$$
\frac{d N_{e^{+}}}{d E_{e^{+}}}= \begin{cases}\delta\left(E_{e^{+}}-m_{\chi}\right)+\left(\alpha_{\mathrm{em}} / 2 \pi\right)\left[3 \delta\left(E_{e^{+}}-m_{\chi}\right) / 2+\right. & \\ \left.\left(1+y^{2}\right) \ln \left(4 m_{\chi}^{2} / m_{e}^{2}\right) /(1-y) m_{\chi}\right] & \text { for } \chi \chi \rightarrow e^{+} e^{-}, \\ \left(\alpha_{\mathrm{em}} / \pi\right) A \exp \left[-\left(A_{1} y+A_{2} y^{2}\right)\right]+B_{1}+B_{2} y & \text { for } \chi \chi \rightarrow \mu^{+} \mu^{-}, \\ \left(1 / m_{\chi}\right)\left(\exp \left[A_{0}^{-}+A_{1}^{-} y+A_{2}^{-} y^{2}+A_{3}^{-} y^{3}\right]+\right. & \\ \left.\exp \left[A_{0}^{+}+A_{1}^{+} y+A_{2}^{+} y^{2}+A_{3}^{+} y^{3}+A_{4}^{+} y^{4}+A_{5}^{+} y^{5}\right]\right) & \text { for } \quad \chi \chi \rightarrow \tau^{+} \tau^{-},\end{cases}
$$

where $\alpha_{\mathrm{em}}$ is the fine-structure constant computed at a scale equal to $2 m_{\chi}, m_{e}=0.511 \mathrm{MeV}$ is the $e^{-}$mass and $0<y=E_{e^{+}} / m_{\chi} \leq 1$. The infrared singularity encountered for $\chi \chi \rightarrow e^{+} e^{-}$and $y=1$ is handled as described in Ref. [53]. For the $\chi \chi \rightarrow \mu^{+} \mu^{-}$mode, we take $J=\tilde{J}\left(m_{\chi} / 0.5 \mathrm{TeV}\right)^{\delta_{J}}$ with $J=A, A_{1}, A_{2}, B_{1}, B_{2}$ and

$$
\begin{aligned}
& \left(\tilde{A}, \tilde{A}_{1}, \tilde{A}_{2}, \tilde{B}_{1}, \tilde{B}_{2}\right)=(-0.296635,2.65121,14.8445,0.0042505,-0.00427157) \\
& \left(\delta_{A}, \delta_{A_{1}}, \delta_{A_{2}}, \delta_{B_{1}}, \delta_{B_{2}}\right)=(-1.01424,0.017198,-0.0107585,-0.999819,-0.999819) .
\end{aligned}
$$

We checked that the parametrization above gives results quite similar to those obtained using the simpler parametrization suggested in Ref. [55]. For the $\chi \chi \rightarrow \tau^{+} \tau^{-}$mode, we take [55]

$$
\begin{aligned}
& \left(A_{0}^{-}, A_{1}^{-}, A_{2}^{-}, A_{3}^{-}\right)=(0.951,-18.083,15.79,-15.575), \\
& \left(A_{0}^{+}, A_{1}^{+}, A_{2}^{+}, A_{3}^{+}, A_{4}^{+}, A_{5}^{+}\right)=(2.783,-22.942,82.595,-193.748,223.389,-97.716) .
\end{aligned}
$$

Also, $I\left(\lambda_{D}\right)$ is the dimensionless halo function which fully encodes the galactic astrophysics with $\lambda_{D}\left(E, E^{\prime}\right)$ the diffusion length from energy $E$ to energy $E^{\prime}$ which is given by

$$
\lambda_{D}^{2}=4 K_{0} t_{E}\left[\frac{\left(E^{\prime} / \mathrm{GeV}\right)^{\delta-1}-(E / \mathrm{GeV})^{\delta-1}}{\delta-1}\right] .
$$

To compute $I\left(\lambda_{D}\right)$ we employ the semi-empirical function proposed in Ref. [50, 58]. Namely,

$$
I\left(\lambda_{D}\right)=a_{0}+a_{1} \tanh \left(\frac{b_{1}-l}{c_{1}}\right)\left[a_{2} \exp \left(-\frac{\left(l-b_{2}\right)^{2}}{c_{2}}\right)+a_{3}\right] \text { with } l=\log \left(\frac{\lambda_{D}}{\mathrm{kpc}}\right) .
$$

The involved in Eqs. (4.3) and (4.4) constants [50] depend on the CDM distribution and the propagation model that we consider. As we emphasize in Sec. 5.1.4, the constraint from the $\gamma$ CRs enforces us to adopt the isothermal halo profile [59] which weakens the relative restrictions. Note, however, that our results on $\Phi_{e^{+}}^{\chi \chi}$ are quite close to those that we would had obtained if we had used the NFW halo [60] profile - c.f. Ref. [31, 50]. We also use the MED propagation model for $\chi \chi \rightarrow e^{-} e^{+}$and $\chi \chi \rightarrow \mu^{-} \mu^{+}$and the MIN (M2) model for $\chi \chi \rightarrow \tau^{-} \tau^{+}$. These choices provide the bets fits to the combined experimental data - c.f. Ref. [58, 61]. Note that only these two propagation models are consistent [49] with the observed boron-to-carbon ratio in the CR flux. Therefore, we use [50]

$$
\begin{aligned}
& \left(a_{0}, a_{1}, a_{2}, a_{3}, b_{1}, b_{2}, c_{1}, c_{2}, K_{0}, \delta\right)= \\
& \begin{cases}\left(0.495,0.629,0.137,0.784,0.766,0.55,0.193,0.296,0.0112 \mathrm{kpc}^{2} / \mathrm{My}, 0.7\right) & (\mathrm{MED}), \\
\left(0.5,0.903,-0.449,0.557,0.096,192.8,0.210,33.91,0.00595 \mathrm{kpc}^{2} / \mathrm{My}, 0.55\right) & \text { (MIN) }\end{cases}
\end{aligned}
$$

We explicitly verified that the numerically fitted function in Eq. (4.4) reproduces quite accurately and fast enough the results obtained by performing numerically the relevant integrations presented 
in the earlier formulae of Ref. [49]. Moreover, the formalism of Ref. [50] overcomes successfully the mismatching problem (in the numerical integration) which is mentioned in Ref. [62].

In order to calculate the total fluxes, we also have to estimate the background $e^{ \pm}$fluxes. In our study, we take into account the fluxes of (i) secondary $e^{+}\left(\Phi_{e^{+}}^{\mathrm{sec}}\right)$ produced by collisions between primary protons and interstellar medium in our galaxy (ii) primary $e^{-}\left(\Phi_{e^{-}}^{\text {prim }}\right)$ presumably produced in supernova remnants and (iii) secondary $e^{-}\left(\Phi_{e^{-}}^{\mathrm{sec}}\right)$ produced by spallation of CRs in the interstellar medium. These fluxes are commonly parameterized as [48]

$$
\begin{aligned}
\Phi_{e^{+}}^{\mathrm{sec}} & =\frac{4.5(E / \mathrm{GeV})^{0.7}}{1+650(E / \mathrm{GeV})^{2.3}+1500(E / \mathrm{GeV})^{4.2}}, \\
\Phi_{e^{-}}^{\mathrm{prim}} & =\frac{0.16(E / \mathrm{GeV})^{-1.1}}{1+11(E / \mathrm{GeV})^{0.9}+3.2(E / \mathrm{GeV})^{2.15}}, \\
\Phi_{e^{-}}^{\mathrm{sec}} & =\frac{0.7(E / \mathrm{GeV})^{0.7}}{1+110(E / \mathrm{GeV})^{1.5}+600(E / \mathrm{GeV})^{2.9}+580(E / \mathrm{GeV})^{4.2}},
\end{aligned}
$$

in units $\mathrm{GeV}^{-1} \mathrm{~cm}^{-2} \mathrm{~s}^{-1} \mathrm{sr}^{-1}$. With these backgrounds, the total $e^{ \pm}$fluxes read

$$
\Phi_{e^{+}}=\Phi_{e^{+}}^{\chi \chi}+\Phi_{e^{+}}^{\mathrm{sec}} \text { and } \Phi_{e^{-}}=\Phi_{e^{-}}^{\chi \chi}+c_{e^{-}} \Phi_{e^{-}}^{\mathrm{prim}}+\Phi_{e^{-}}^{\mathrm{sec}}
$$

where $\Phi_{e^{-}}^{\chi \chi}=\Phi_{e^{+}}^{\chi \chi}$ and $c_{e^{-}} \simeq(0.6-0.8)$ is a normalization factor. We take $c_{e^{-}}=0.6\left[c_{e^{-}}=0.7\right]$ so that our flux calculation is consistent with the ATIC [Fermi-LAT] data in the low energy range of $(20-70) \mathrm{GeV}[58]$.

\subsection{Fitting the PAMELA and ATIC or Fermi-LAT Data}

Using the fluxes defined above, we can evaluate the observable quantities and compare them with the experimental outputs. In order to qualify our fittings to the experimental data, we perform a $\chi^{2}$ analysis. In particular, we define the $\chi^{2}$ variables as [54, 58, 62]

$$
\chi_{A}^{2}=\sum_{i=1}^{N_{A}} \frac{\left(F_{A i}^{\mathrm{obs}}-F_{A i}^{\mathrm{th}}\right)^{2}}{\left(\Delta F_{A i}^{\mathrm{obs}}\right)^{2}}, \text { with } F_{A}= \begin{cases}\Phi_{e^{+}} /\left(\Phi_{e^{+}}+\Phi_{e^{-}}\right) & \text {and } N_{A}=7 \text { for } A=1, \\
E_{e^{+}}^{3}\left(\Phi_{e^{+}}+\Phi_{e^{-}}\right) & \text {and } N_{A}=\left\{\begin{array}{lll}
21 & \text { for } A=2, \\
26 & \text { for } A=3,
\end{array}\right.\end{cases}
$$

where $A=1,2,3$ stands for the PAMELA [24], ATIC [25] or Fermi-LAT [30] data respectively which are considered as independent sets. The index $i$ runs over the data points of each experiment $A$, the superscript "obs" ["th"] refers to measured [theoretically predicted] quantities whereas $\Delta F^{\text {obs }}$ means error in the experimentally observable $F . N_{A}$ is the number of data points considered from the experiment $A$. Note that, from the PAMELA data-set, we use $[58,54]$ only the 7 data points above $9.1 \mathrm{GeV}$ where the effect of solar modulation is expected to be small. In our analysis we take into account only the vertical errors. We also conservatively combine, independently for each data-point, in quadrature statistical and systematic errors released from Fermi LAT [30].

In Fig. 7 we show the predicted observable quantities compared to the experimental data as a function of the $e^{+}$energy $E_{e^{+}}$, assuming $\chi$ annihilating to $e^{+} e^{-}$(dot-dashed lines), $\mu^{+} \mu^{-}$(dashed lines) or $\tau^{+} \tau^{-}$(dotted lines). We use the best-fit $\left(m_{\chi},\langle\sigma v\rangle\right)$ 's obtained from minimization of $\chi_{1}^{2}+\chi_{2}^{2}$ $\left[\chi_{1}^{2}+\chi_{3}^{2}\right]$ in Fig. 7-(a $\left.a_{1}\right)$ and $\left(a_{2}\right)$ [Fig. 7- $\left(b_{1}\right)$ and $\left(b_{2}\right)$. Since ATIC and Fermi-LAT data are not consistent with each other, we do not combine them but present results using only either of the two. The relevant $\left(m_{\chi},\langle\sigma v\rangle\right)$ 's can be read in the Table 2 together with the corresponding $\chi^{2} /$ d.o.f, where 


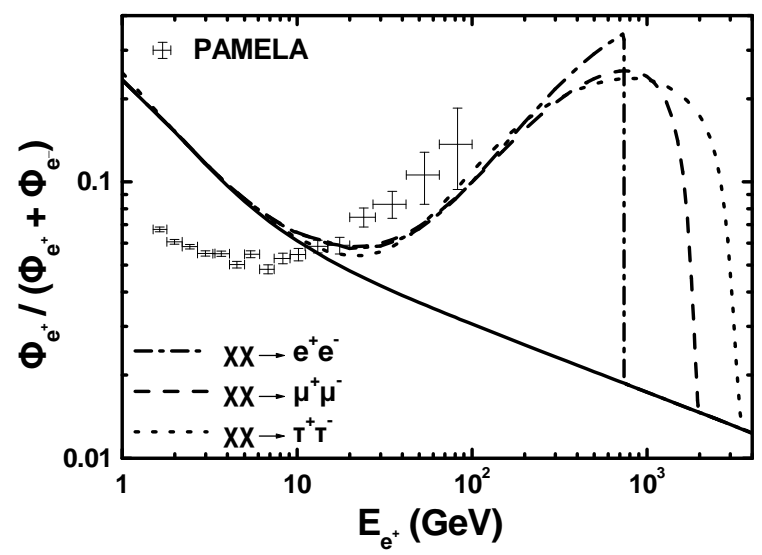

$\left(a_{1}\right)$

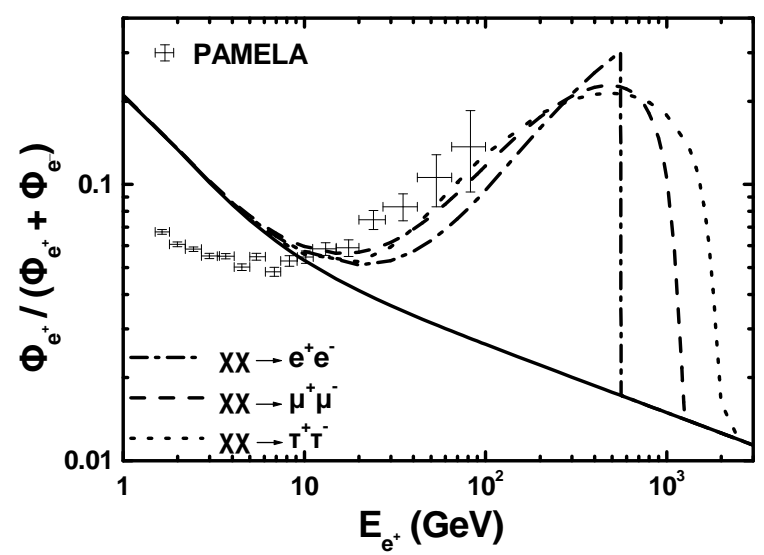

$\left(b_{1}\right)$

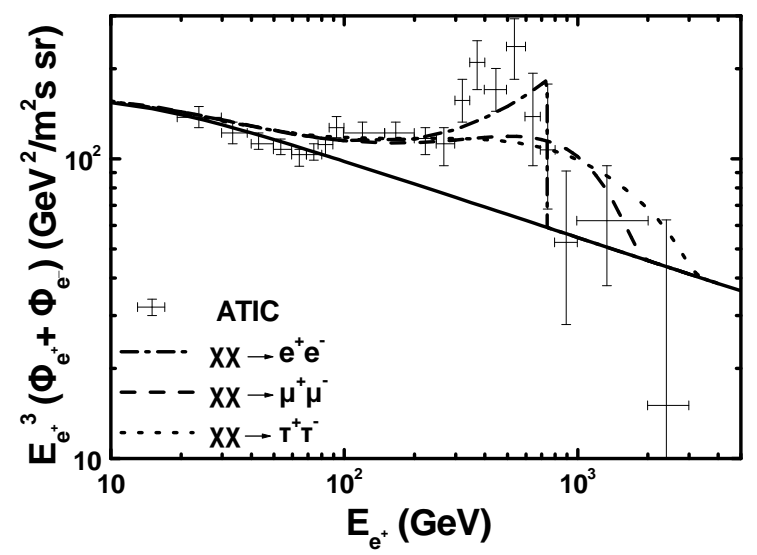

$\left(a_{2}\right)$

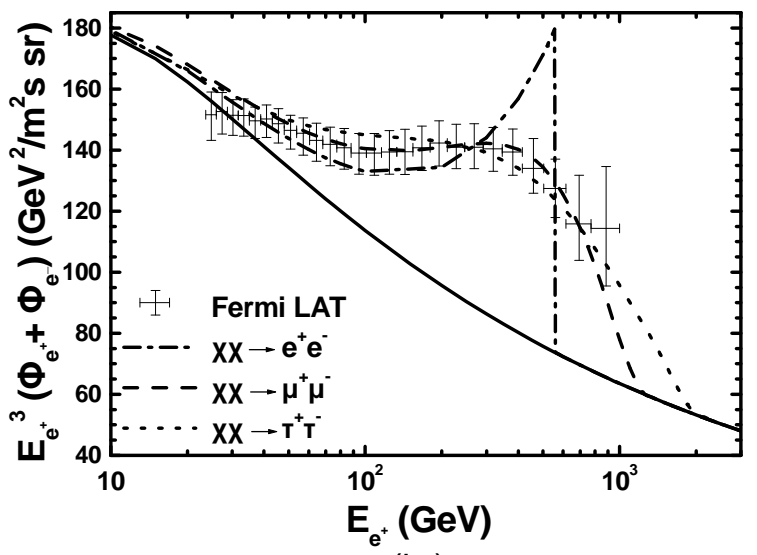

$\left(b_{2}\right)$

Figure 7: The $e^{+}$-flux fraction $\left(\mathrm{a}_{1}\right.$ and $\left.\mathrm{b}_{1}\right)$ and the total $e^{-}$and $e^{+}$flux times $E_{e^{+}}^{3}\left(\mathrm{a}_{2}\right.$ and $\left.\mathrm{b}_{2}\right)$ as a function of $E_{e^{+}}$, with $E_{e^{+}}$being the $e^{+}$energy. We use the best-fit points $\left(m_{\chi},\langle\sigma v\rangle\right)$, indicated in Table 2, for the PAMELA and ATIC data ( $\mathrm{a}_{1}$ and $\left.\mathrm{a}_{2}\right)$ or the PAMELA and Fermi-LAT data $\left(\mathrm{b}_{1}\right.$ and $\left.\mathrm{b}_{2}\right)$, assuming $\chi$ annihilating into $e^{+} e^{-}$(dot-dashed lines), $\mu^{+} \mu^{-}$ (dashed lines) or $\tau^{+} \tau^{-}$(dotted lines). The background fluxes are denoted by solid lines and are computed for $c_{e^{-}}=0.6$ $\left(\mathrm{a}_{1}\right.$ and $\left.\mathrm{a}_{2}\right)$ or $c_{e^{-}}=0.7\left(\mathrm{~b}_{1}\right.$ and $\left.\mathrm{b}_{2}\right)$. The data from PAMELA $\left(\mathrm{a}_{1}, \mathrm{~b}_{1}\right)$, ATIC $\left(\mathrm{a}_{2}\right)$ and Fermi-LAT $\left(\mathrm{b}_{2}\right)$ experiments are also shown (an additional uncertainty from the Fermi-LAT energy scale, which can shift all the points by $5 \%$ (up) to $10 \%$ (down) is not shown). Recall that we adopt the isothermal halo profile and the MED [MIN] propagation model for $\chi \chi \rightarrow e^{+} e^{-}$and $\chi \chi \rightarrow \mu^{+} \mu^{-}\left[\chi \chi \rightarrow \tau^{+} \tau^{-}\right]$.

d.o.f denotes the number of degrees of freedom involved in our fits which is equal to $N_{1}+N_{2}-2=26$ $\left[N_{1}+N_{3}-2=31\right]$ for PAMELA and ATIC [PAMELA and Fermi-LAT] data ( 2 is the number of the fitting variables, $m_{\chi}$ and $\langle\sigma v\rangle$ ). As can be deduced from Table 2, an exceptionally good fit to PAMELA and Fermi-LAT data arises for $\chi$ annihilating to $\mu^{+} \mu^{-}$, whereas in most other cases the fits are rather poor since we get just $\chi^{2} /$ d.o.f $\simeq 2.5-3$ for 28 or 33 data points. Better fits can be probably attained under the assumption that $\chi$ 's both annihilate and decay, as pointed out in Ref. [58]. Note finally that, the $\chi$ annihilation into $e^{+} e^{-}$is strongly disfavored [63] by Fermi-LAT data since it predicts a spectrum with a too sharp end-point.

In Table 2 we also list the maximal $\langle\sigma v\rangle,\langle\sigma v\rangle_{\max }$, allowed by Eq. (5.4) (see Sec. 5.1.3) and the resulting $\left.\Omega_{\chi} h^{2}\right|_{\text {SC }}$ 's. It is remarkable that $\left.\Omega_{\chi} h^{2}\right|_{\text {SC }}$ turns out to be well below the range of Eq. (1.2) implied by the CDM considerations. As a consequence, the SC can not be consistent with the interpretation of the $e^{ \pm}$-CR anomalies via $\chi$ annihilation, unless we invoke an enhancement 


\begin{tabular}{|c|c|c|c|c|c|c|}
\hline FigURE & $\begin{array}{l}\text { ANNIHILATION } \\
\text { Mode }\end{array}$ & $\begin{array}{c}\left.\chi^{2}\right|_{\min } / \\
\text { d.o.f }\end{array}$ & $\begin{array}{l}m_{\chi} / \\
\mathrm{TeV}\end{array}$ & $\begin{array}{c}\langle\sigma v\rangle / \\
10^{-7} \mathrm{GeV}^{-2}\end{array}$ & $\begin{array}{c}\langle\sigma v\rangle_{\max } / \\
10^{-7} \mathrm{GeV}^{-2}\end{array}$ & $\begin{array}{c}\left.\Omega_{\chi} h^{2}\right|_{\mathrm{SC}} / \\
10^{-4}\end{array}$ \\
\hline 7- $\left(\mathrm{a}_{1}\right)$ and $\left(\mathrm{a}_{2}\right)$ & $\chi \chi \rightarrow e^{+} e^{-}$ & $67 / 26$ & 0.74 & 7 & 3.3 & 3.85 \\
\hline 7-( $\left(\mathrm{a}_{1}\right)$ and $\left(\mathrm{a}_{2}\right)$ & $\chi \chi \rightarrow \mu^{+} \mu^{-}$ & $76 / 26$ & 2 & 28.6 & 26 & 0.97 \\
\hline 7-( $\left(\mathrm{a}_{1}\right)$ and $\left(\mathrm{a}_{2}\right)$ & $\chi \chi \rightarrow \tau^{+} \tau^{-}$ & $79 / 26$ & 3.5 & 143 & 20 & 0.2 \\
\hline $7-\left(b_{1}\right)$ and $\left(b_{2}\right)$ & $\chi \chi \rightarrow e^{+} e^{-}$ & $75 / 31$ & 0.5595 & 4.6 & 2.5 & 5.8 \\
\hline 7-( $\left.b_{1}\right)$ and $\left(b_{2}\right)$ & $\chi \chi \rightarrow \mu^{+} \mu^{-}$ & $24 / 31$ & 1.28 & 19.5 & 16.5 & 1.4 \\
\hline 7- $\left(\mathrm{a}_{1}\right)$ and $\left(\mathrm{a}_{2}\right)$ & $\chi \chi \rightarrow \tau^{+} \tau^{-}$ & $33 / 31$ & 2.1 & 94.6 & 28.2 & 0.3 \\
\hline
\end{tabular}

TABLE 2: The best-fit points $\left(m_{\chi},\langle\sigma v\rangle\right)$ for the PAMELA and ATIC data (used in Fig. 7-( $\left.\mathrm{a}_{1}\right)$ and $\left(\mathrm{a}_{2}\right)$ ) or the PAMELA and Fermi-LAT data (used in Fig. 7- $\left(\mathrm{b}_{1}\right)$ and $\left.\left(\mathrm{b}_{2}\right)\right)$ for each annihilation channel of $\chi$. Reported are also the corresponding $\left.\chi^{2}\right|_{\min }$ /d.o.f., the maximal $\langle\sigma v\rangle,\langle\sigma v\rangle_{\max }$, allowed by Eq. (5.4) and the resulting $\Omega_{\chi} h^{2}$ in the $S C,\left.\Omega_{\chi} h^{2}\right|_{\mathrm{SC}}$.

mechanism of $\langle\sigma v\rangle$ at present [26, 27]. In other words, some $\Delta \Omega_{\chi}$ is necessitated in order to reconcile the best-fit $\left(m_{\chi},\langle\sigma v\rangle\right)$ 's with Eq. (1.2). Moreover, we observe that the bound of Eq. (5.4) (which turns out to be the most restrictive of all the others presented in Sec. 5.1) is violated in all cases. This violation is softer [stronger] in the case where $\chi$ 's annihilate to $\mu^{+} \mu^{-}\left[\tau^{+} \tau^{-}\right]$. We observe, also, that for $\chi \chi \rightarrow \tau^{+} \tau^{-}, m_{\chi}$ 's and $\langle\sigma v\rangle$ 's are pushed to larger values than those needed for $\chi \chi \rightarrow \mu^{+} \mu^{-}$and the $m_{\chi}$ 's and $\langle\sigma v\rangle$ 's used in the latter case are higher than those used for $\chi \chi \rightarrow e^{+} e^{-}$. As shown in the next section, best-fit $\left(m_{\chi},\langle\sigma v\rangle\right)$ 's consistent with all the available constraints can be achieved for $\chi$ 's annihilating to $e^{+} e^{-}$or $\mu^{+} \mu^{-}$.

\section{Restrictions in the $m_{\chi}-\langle\sigma v\rangle$ Plane}

To systematize our approach, we need to delineate in the $m_{\chi}-\langle\sigma v\rangle$ plane the regions which are favored at $95 \%$ c.l. by the various experimental data on the $e^{ \pm}$-CRs. In particular, we consider $[58,54]$ regions favored by PAMELA data only, PAMELA and ATIC data or PAMELA and Fermi LAT data. Since we have two independent parameters, $m_{\chi}$ and $\langle\sigma v\rangle$ these regions can be determined imposing the condition [54]

$$
\left.\chi^{2} \lesssim \chi^{2}\right|_{\min }+6 \text { with } \chi^{2}= \begin{cases}\chi_{1}^{2} & \text { for PAMELA, } \\ \chi_{1}^{2}+\chi_{2}^{2} & \text { for PAMELA and ATIC, } \\ \chi_{1}^{2}+\chi_{3}^{2} & \text { for PAMELA and Fermi LAT }\end{cases}
$$

where $\left.\chi^{2}\right|_{\min }$ can be extracted numerically by minimization of $\chi^{2}$ w.r.t $m_{\chi}$ and $\langle\sigma v\rangle$. On the other hand, the interpretation of the data on $e^{ \pm}$-CRs in terms of $\chi$ annihilation can be viable if it can become consistent with a number of phenomelogical constraints. In Sec. 5.1 we summarize these constraints and in Sec. 5.2 we examine if they can be reconciled with the regions favored by the data on $e^{ \pm}$-CRs.

\subsection{Imposed Constraints}

Though $n_{\chi} / s$ in Eq. (3.1) stays essentially unchanged for $\tau>\tau_{\mathrm{f}}$, residual annihilations of $\chi$ 's occur up to the present with several cosmological consequences besides the possible interpretation 
of the data on $e^{ \pm}$-CRs. Recently, important upper bounds on $\langle\sigma v\rangle$ have been reported and are summarized below for the three exemplary $\chi$ annihilation modes considered in our investigation. The well-known unitarity constraint is also taken into account.

5.1.1 UnITARITY CONSTRAINT. Using partial-wave unitarity $[23,65]$ an upper limit, particularly relevant for $m_{\chi}>2 \mathrm{TeV}$, on $\langle\sigma v\rangle$ can be derived as a function of $m_{\chi}$, i.e.,

$$
\langle\sigma v\rangle \leq 8 \pi \mathrm{GeV}^{-2}\left(\frac{m_{\chi}}{1 \mathrm{GeV}}\right)^{-2} .
$$

5.1.2 BBN Constraint. During BBN, the $\chi$ annihilations inject an amount of energetic particles which is proportional to $\langle\sigma v\rangle$ and may strongly alter $[61,64]$ the abundances of the light elements. Ruining the successful predictions of the BBN can be avoided if we impose an upper bound on $\langle\sigma v\rangle$ which, however, depends on the identity of the products of the annihilation of $\chi$ 's. Taking into account the most up-to-date analysis of Ref. [61] we demand:

$$
\langle\sigma v\rangle \leq 3 \cdot 10^{-5} \mathrm{GeV}^{-2} \frac{2 m_{\chi}}{E_{\mathrm{vis}}} \frac{m_{\chi}}{1 \mathrm{TeV}} \text { where } \frac{E_{\mathrm{vis}}}{m_{\chi}}=\left\{\begin{array}{l}
2 \text { for } \chi \chi \rightarrow e^{+} e^{-}, \\
0.7 \text { for } \chi \chi \rightarrow \mu^{+} \mu^{-}, \\
0.62 \text { for } \chi \chi \rightarrow \tau^{+} \tau^{-}
\end{array}\right.
$$

with $E_{\mathrm{vis}}$ being the total visible energy of the produced particles in the $\chi$ annihilation.

5.1.3 CMB Constraint. The $\chi$ annihilations may have $[32,33]$ an impact on the ionization state of the baryonic gas at recombination and therefore on the CMB angular spectra. Consistency with the WMAP5 data [1] dictates [33] at 95\% c.l. (see, also, Ref. [34]):

$$
\langle\sigma v\rangle \leq \frac{3.1 \cdot 10^{-7} \mathrm{GeV}^{-2}}{f} \frac{m_{\chi}}{1 \mathrm{TeV}} \text { where } f \simeq\left\{\begin{array}{l}
0.7 \text { for } \chi \chi \rightarrow e^{+} e^{-} \\
0.24 \text { for } \chi \chi \rightarrow \mu^{+} \mu^{-} \\
0.23 \text { for } \chi \chi \rightarrow \tau^{+} \tau^{-}
\end{array}\right.
$$

is the deposited power fraction which expresses the efficiency of the coupling between the annihilation products and the photon-baryon fluid at $z \sim 1000$. It is expected that forthcoming experiments will impose [33] even more stringent bounds on $\langle\sigma v\rangle$. Note, in passing, that the presence of $q$ in the QKS does not affect recombination (which occurs at $\tau_{\text {rec }} \simeq-7$ ) since $\Omega_{q}\left(\tau_{\text {rec }}\right.$ ) is safely suppressed provided that Eq. (2.28) is fulfilled.

5.1.4 Constraint from the $\gamma$-Cosmic Rays. The $\chi$ annihilation in the galactic center yields sizeable amount of $\gamma$-CRs, through the cascade decay of the annihilation products and/or bremsstrahlung processes. Comparing the relevant $\gamma$-CR flux with the H.E.S.S observations [66] we can further restrict [31] $\langle\sigma v\rangle$ as a function of $m_{\chi}$ for the two chosen annihilation channels. However, this restriction significantly depends on the CDM halo profile. Adopting the cored isothermal CDM profile [59], which assures the less restrictive version of this constraint, we graphically extract the upper bound on $\langle\sigma v\rangle$ from the plots of Ref. [31]. To have a feeling of the strength of this constraint we can give some rough estimations:

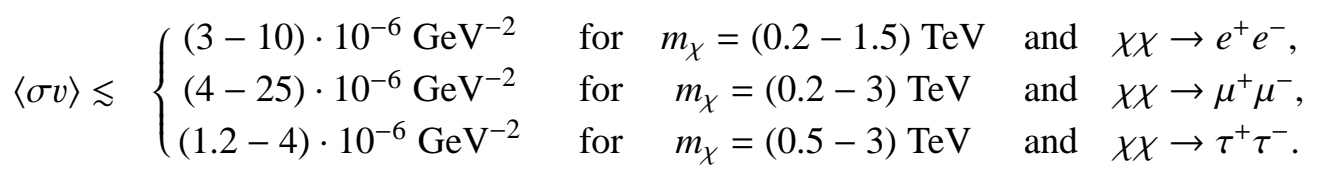

Alternatively this constraint can be evaded for every CDM profile, by allowing the $\chi$-annihilation products to be long lived, as pointed out in Ref. [54]. 
Complementary constraints on the $\chi$ annihilation can be imposed comparing the findings of EGRET satellite [67] with the diffuse (secondary) $\gamma$-CR fluxes, which would be produced [68] by inverse Compton scatterings on interstellar photons of the energetic $e^{ \pm}$generated by the $\chi$ annihilation in the galactic halo. However, these bounds are expected [68] to be weaker than the ones imposed by the high energy $\gamma$-CRs mentioned in Sec. 5.1.4 and are not included in our analysis. Similar arguments are [69] also valid for the neutrinos generated from the $\chi$ annihilation in the galactic center, though the dependence on the CDM profile is weaker.

\subsection{Results}

Constructing the preferred areas by the various experimental data and taking into account the constraints quoted in Sec. 5.1 we can check the viability of the interpretation of the anomalies on $e^{ \pm}$-CR fluxes in terms of the $\chi$ annihilation. In Fig. 8 we consider the mode $\chi \chi \rightarrow \tau^{+} \tau^{-}$whereas in Fig. 9- $\left(\mathrm{a}_{1}\right),\left(\mathrm{b}_{1}\right)$ and $\left(\mathrm{c}_{1}\right)$ [Fig. 9- $\left(\mathrm{a}_{2}\right),\left(\mathrm{b}_{2}\right)$ and $\left(\mathrm{c}_{2}\right)$ ] we assume that $\chi$ 's annihilate into $e^{+} e^{-}\left[\mu^{+} \mu^{-}\right]$.

In Fig. 8 and 9 we delineate the regions preferred at 95\% c.l. by PAMELA data (black and red sparse hatched areas), PAMELA and ATIC data (dense black hatched areas) and PAMELA and Fermi-LAT data (dense red hatched areas), by imposing the condition of Eq. (5.1). In the black [red] hatched areas the backgrounds fluxes are normalized setting $c_{e^{-}}=0.6\left[c_{e^{-}}=0.7\right]$ in Eq. (4.7). Evidently, the PAMELA data do not prefer any $m_{\chi}$ since it does not show any peak structure whereas Fermi-LAT data disfavors the mode $\chi \chi \rightarrow e^{+} e^{-}$since the spectrum from such a channel is too peaked to reproduce data. We also remark that the regions derived by the joint analysis of two data-sets are rather limited - c.f. Ref. [31, 34, 54, 63]. We consider the latter results as more reliable, since even when we fit only the PAMELA data, the data points with low $E_{e^{+}}$from ATIC/Fermi LAT are involved, in order to normalize [54] the background fluxes.

In Fig. 8 and 9 drawn is also the upper bound from Eqs. (5.2), (5.3) and (5.4), denoted by a black dotted, solid and dashed line respectively and this from the constraint of Sec. 5.1.4, depicted by a dot-dashed line. Obviously acceptable are the regions mainly below the dashed curves, since the bound of Eq. (5.4) is the most restrictive from the others. The bound of Eq. (5.2) cuts out some slices of the parameter space for $\chi \chi \rightarrow \mu^{+} \mu^{-}$and $\chi \chi \rightarrow \tau^{+} \tau^{-}$and large $m_{\chi}$ 's. We easily conclude that the explanation of the experimental anomalies via the annihilation mode:

- $\chi \chi \rightarrow e^{+} e^{-}$is just marginally consistent with Eq. (5.4) - see Fig. 9- $\left(\mathrm{a}_{1}\right),\left(\mathrm{b}_{1}\right)$ and $\left(\mathrm{c}_{1}\right)$. Indeed, we observe that just a minor portion of the area favored by PAMELA at $95 \%$ c.l. is allowed by Eq. (5.4) whereas the regions preferred at 95\% c.l. from both combinations of PAMELA and ATIC or PAMELA and Fermi-LAT data are entirely excluded from the bounds of Eq. (5.4).

- $\chi \chi \rightarrow \mu^{+} \mu^{-}$can be reconciled - c.f. Ref. [23, 31, 54, 61] - with the various constraints - see Fig. 9- $\left(a_{2}\right),\left(b_{2}\right)$ and $\left(c_{2}\right)$. Namely, we notice that sizable slices of the regions favored by PAMELA lie below the bound of Eq. (5.4). Moreover, very close to or even lower than this limit we find portions of the favored regions at $95 \%$ c.l. by the PAMELA and ATIC or Fermi-LAT data.

- $\chi \chi \chi \tau^{+} \tau^{-}$is inconsistent - c.f. Ref. [31] - with both Eq. (5.4) and Eq. (5.5) at 95\% c.l. since all the regions favored by the experimental data lie entirely above the bounds above see Fig. 8. Violation of the bound of Eq. (5.2) in a sizable fraction of these regions is observed too. Because of this fact, we below concentrate on the other two annihilation modes of $\chi$ 's. 


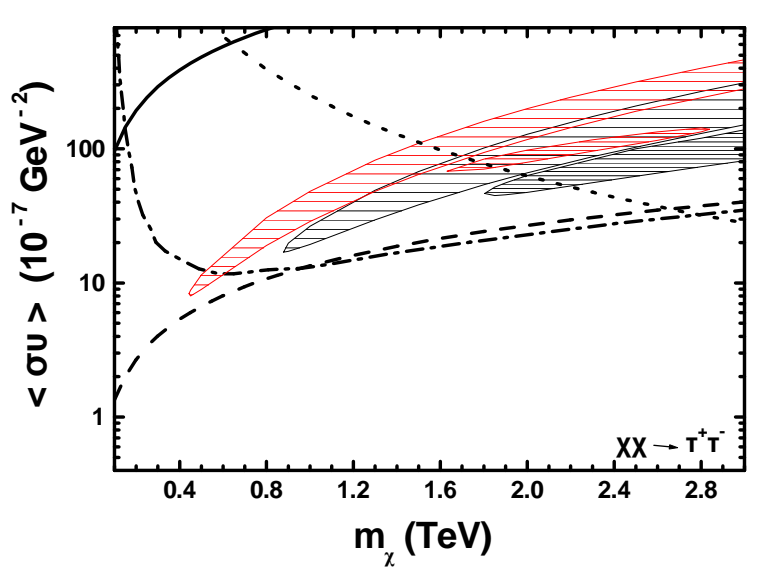

Figure 8: Restrictions in the $m_{\chi}-\langle\sigma v\rangle$ plane for $\chi$ 's annihilating into $\tau^{+} \tau^{-}$. The sparse black [red] hatched areas are preferred at $95 \%$ c.l. by the PAMELA data for $c_{e^{-}}=0.6\left[c_{e^{-}}=0.7\right]$ and the dense black [red] hatched areas are preferred at $95 \%$ c.l. by the PAMELA and ATIC [PAMELA and Fermi-LAT] data. Regions above the black solid, dashed, dotted and dot-dashed lines are ruled out by the upper bounds on $\langle\sigma v\rangle$ from Eq. (5.3), (5.4), (5.2) and Sec. 5.1 .4 correspondingly.

Fixing the parameters related to the LRS [QKS] to some representative values - consistent with Sec. 2.2.2 [Sec. 2.3.2] -, we can display in the $m_{\chi}-\langle\sigma v\rangle$ plane, as in Fig. 9, regions (light gray shaded) confronted with Eq. (1.2). The gray dashed [dotted] lines correspond to Eq. (1.2b) [Eq. (1.2a)], whereas the gray solid lines are obtained by fixing $\Omega_{\chi} h^{2}$ to its central value in Eq. (1.2). Fig. 9- $\left(a_{1}\right)$ and $\left(a_{2}\right)$ are devoted to the LRS whereas Fig. 9- $\left(b_{1}\right),\left(b_{2}\right),\left(c_{1}\right)$ and $\left(c_{2}\right)$ analyze the QKS.

For the LRS, we confine ourselves to some combinations of parameters which assure a sufficient coexistence of non-TP and TP, since non-TP alone is obviously - see Eq. (3.7) $-\langle\sigma v\rangle$ independent and therefore can not be properly depicted in the $m_{\chi}-\langle\sigma v\rangle$ plane. We take $\left(c_{\chi \phi}, T_{\mathrm{RH}}\right)=$ $\left(2 \cdot 10^{-6}, 0.1 \mathrm{GeV}\right)$ and $\left(c_{\chi \phi}, T_{\mathrm{RH}}\right)=(1,1 \mathrm{GeV})\left[\left(c_{\chi \phi}, T_{\mathrm{RH}}\right)=\left(10^{-6}, 0.1 \mathrm{GeV}\right)\right.$ and $\left(c_{\chi \phi}, T_{\mathrm{RH}}\right)=$ $(1,0.5 \mathrm{GeV})$ ] in Fig. 9- $\left(\mathrm{a}_{1}\right)$ [Fig. 9- $\left.\left(\mathrm{a}_{2}\right)\right]$. For the QKS, we set throughout $a=0.5, T_{\mathrm{I}}=10^{9} \mathrm{GeV}$. We also take (i) $b=0$ and $\bar{H}_{\mathrm{I}}=6.3 \cdot 10^{53}, 2 \cdot 10^{53}$ or $6.2 \cdot 10^{52}$ resulting to $\Omega_{q}^{\mathrm{BBN}}=0.01,0.001$ or 0.0001 respectively in Fig. $9-\left(\mathrm{b}_{1}\right)$ and $\left(\mathrm{b}_{2}\right)$; (ii) $\bar{H}_{\mathrm{I}}=6.3 \cdot 10^{53}$ and $b=0.15\left[\bar{H}_{\mathrm{I}}=6.2 \cdot 10^{52}\right.$ and $b=0.32]$ resulting to $\Omega_{q}^{\mathrm{BBN}}=0.068$ or $\left[\Omega_{q}^{\mathrm{BBN}}=0.065\right]$ in Fig. $9-\left(\mathrm{c}_{1}\right)$; (iii) $\bar{H}_{\mathrm{I}}=6.3 \cdot 10^{53}$ and $b=0.32\left[\bar{H}_{\mathrm{I}}=2 \cdot 10^{53}\right.$ and $\left.b=0.2\right]$ yielding $\Omega_{q}^{\mathrm{BBN}}=0.19$ or $\left[\Omega_{q}^{\mathrm{BBN}}=0.21\right]$ in Fig. 9- $\left(\mathrm{c}_{2}\right)$. Note that in Fig. 9-( $\left.b_{1}\right)$ and $\left(b_{2}\right)$ we present for the sake of comparison results even for $b=0$, although the tracking behavior of the QKS is not attained in this case - see Sec. 2.3.4.

In all cases, we observe that $\Omega_{\chi} h^{2}$ decreases as $\langle\sigma v\rangle$ increases. This is due to the fact that $\Omega_{\chi} h^{2} \propto 1 /\langle\sigma v\rangle$ as can be deduced from Eq. (3.1) and Eq. (3.8) [Eq. (3.9)] for the LRS [QKS]. For the LRS, as it is clear from these plots, there is a minor slice of the allowed region with $m_{\chi}<0.35 \mathrm{TeV}\left[m_{\chi}<0.8 \mathrm{TeV}\right]$ for $T_{\mathrm{RH}}=0.1 \mathrm{GeV}$ and $c_{\chi \phi}=2 \cdot 10^{-6}\left[c_{\chi \phi}=10^{-6}\right]$ where non-TP is strengthened and our results are almost $\langle\sigma v\rangle$ independent. For the QKS, we also observe that for $\tau_{\mathrm{F}}$ far away from $\tau_{\text {ext }}$ the allowed by Eq. (1.2) for $b \neq 0$ region reaches the one for $b=0-$ with fixed $\bar{H}_{\mathrm{I}}$. However, when $\tau_{\mathrm{F}}$ reaches $\tau_{\text {ext }}, \Omega_{\chi} h^{2}$ decreases (as we explain in Sec. 3.2.2) and so, the required, for obtaining $\Omega_{\chi} h^{2}$ in the range of Eq. (1.2), $\langle\sigma v\rangle$ decreases too. As a consequence, although the allowed by Eq. (1.2) area in Fig. 9-( $\left.\mathrm{c}_{1}\right)$ [Fig. 9- $\left.\left(\mathrm{c}_{2}\right)\right]$ for $\bar{H}_{\mathrm{I}}=6.3 \cdot 10^{53}$ approaches the corresponding area in Fig. 9-( $\left.b_{1}\right)$ [Fig. 9-( $\left.b_{2}\right)$ ] with the same $\bar{H}_{\mathrm{I}}$ and violates the bounds of Eq. (5.4) for low $m_{\chi}$ 's, it becomes compatible with the latter constraint for larger $m_{\chi}$ 's. On the other hand, we observe that there is no such a transition region in the light gray area of Fig. 9- $\left(\mathrm{c}_{2}\right)$. This is, because for $0.1 \leq m_{\chi} / \mathrm{TeV} \leq 3$ we get $31.8 \leq-\tau_{\mathrm{F}} \leq 35.1$ whereas the closest to $\tau_{\mathrm{F}}$ 's, $\tau_{\mathrm{ext}}$ is $\tau_{\mathrm{ext}}=-35.4$ which remains constantly lower than $\tau_{\mathrm{F}}$. Therefore, no reduction of $\Delta \Omega_{\chi}$ occurs for the $m_{\chi}$ 's used in Fig. 9-( $\left.\mathrm{c}_{2}\right)$. 


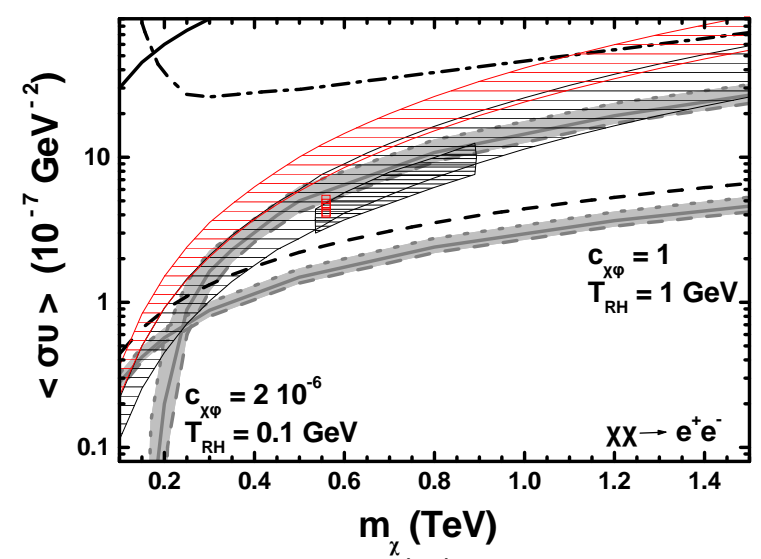

$\left(a_{1}\right)$

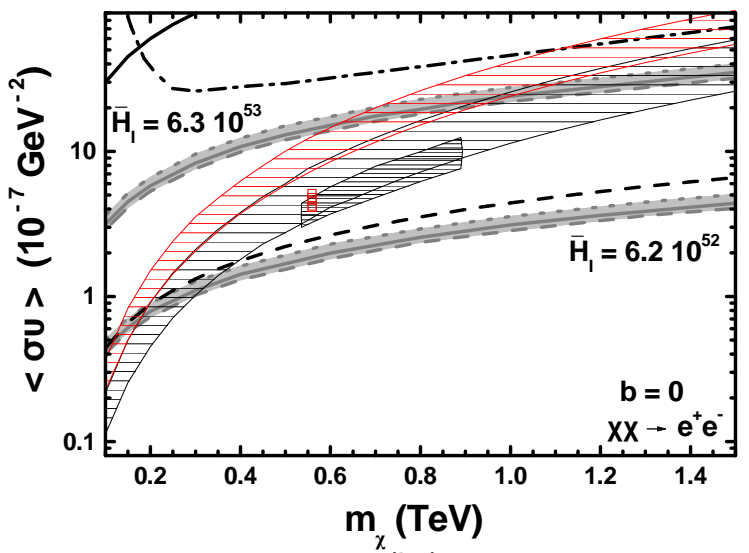

$\left(b_{1}\right)$

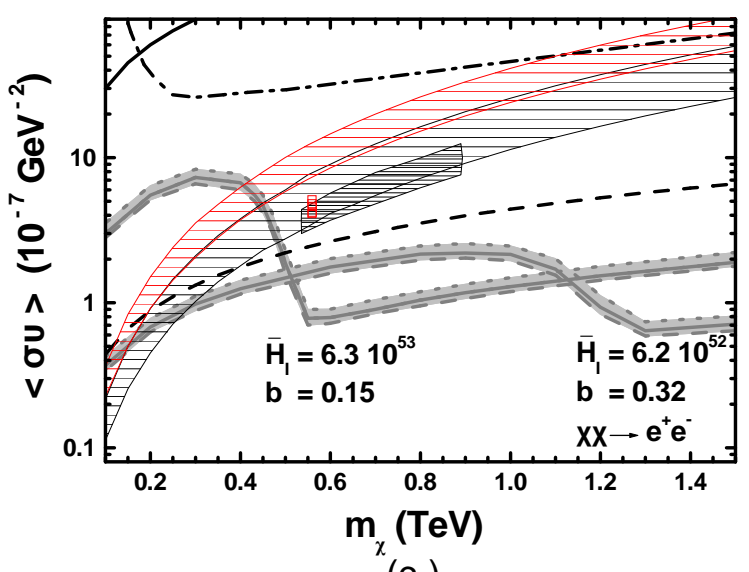

$\left(c_{1}\right)$

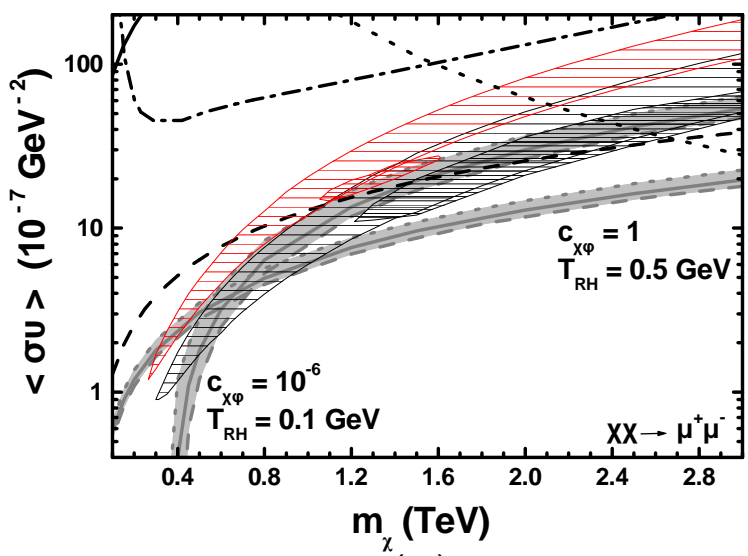

$\left(a_{2}\right)$

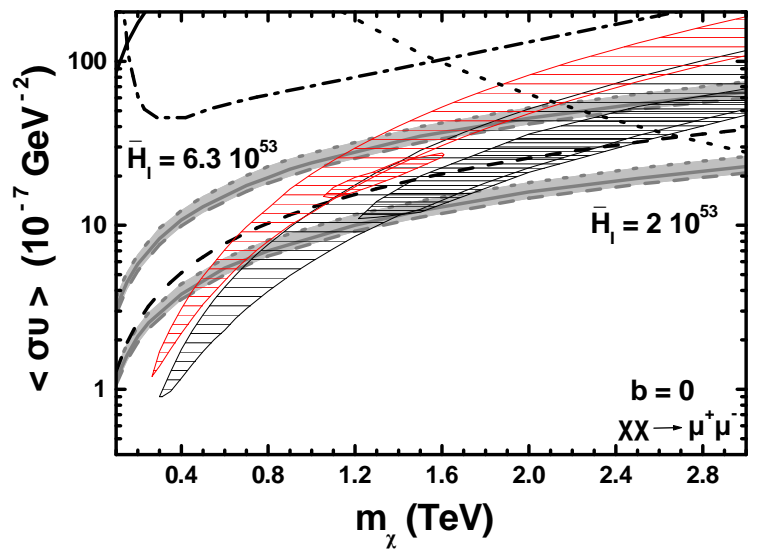

$\left(b_{2}\right)$

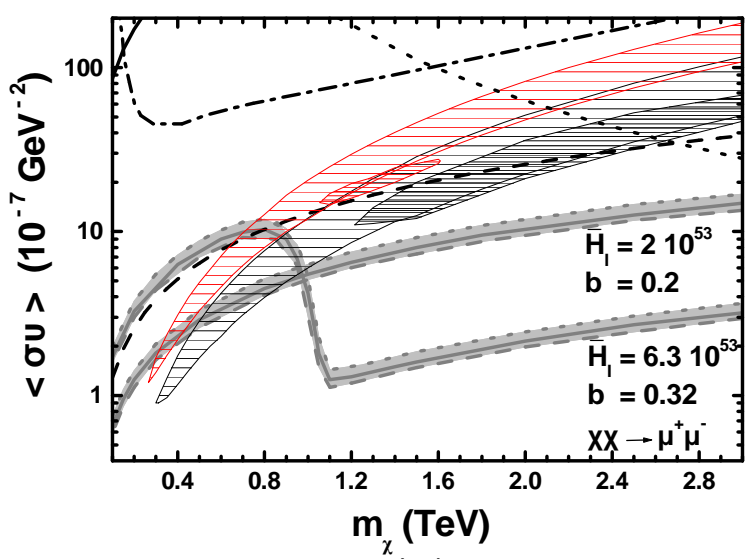

$\left(c_{2}\right)$

FIGURE 9: Restrictions in the $m_{\chi}-\langle\sigma v\rangle$ plane for the LRS $\left(\mathrm{a}_{1}, \mathrm{a}_{2}\right)$ [QKS $\left(\mathrm{b}_{1}, \mathrm{~b}_{2}, \mathrm{c}_{1}, \mathrm{c}_{2}\right)$ taking $a=0.5, T_{\mathrm{I}}=10^{9} \mathrm{GeV}$ ] with various $c_{\chi \phi}$ 's and $T_{\mathrm{RH}}$ 's [ $b$ 's and $\bar{H}_{\mathrm{I}}$ 's], indicated in the graphs, and $\chi$ 's annihilating into $e^{+} e^{-}\left(\mathrm{a}_{1}, \mathrm{~b}_{1}, \mathrm{c}_{1}\right)$ or $\mu^{+} \mu^{-}\left(\mathrm{a}_{2}, \mathrm{~b}_{2}, \mathrm{c}_{2}\right)$. The light gray shaded areas are allowed by Eq. (1.2), the sparse black [red] hatched areas are preferred at $95 \%$ c.l. by the PAMELA data for $c_{e^{-}}=0.6\left[c_{e^{-}}=0.7\right]$ and the dense black [red] hatched areas are preferred at $95 \%$ c.l. by the PAMELA and ATIC [PAMELA and Fermi-LAT] data. Regions above the black solid, dashed, dotted and dot-dashed lines are ruled out by $\begin{aligned} \cdots . \Omega_{x} h^{2} & =0.097 \\ \Omega_{x} h^{2} & =0.11 \\ ---\Omega_{x} h^{2} & =0.12\end{aligned}$ the upper bounds on $\langle\sigma v\rangle$ from Eq. (5.3), (5.4), (5.2) and Sec. 5.1.4 correspondingly. The conventions adopted for the residual lines are also shown. 
As can be concluded from most of the plots of Fig. 9, a simultaneous interpretation of the $e^{ \pm}$-CR anomalies consistently with the requirements of Sec. 5.1 can be achieved in the regions where the gray shaded areas overlap the lined ones below the dashed lines. To clarify further this intriguing conclusion of this paper, it would be interesting to find the best-fit $\left(m_{\chi},\langle\sigma v\rangle\right)$ (for the various combined data-sets) which fulfill all the restrictions imposed in Sec. 5.1. Our results are arranged in Table 3. The listed $\left(m_{\chi},\langle\sigma v\rangle\right)$ 's saturate the bound of Eq. (5.4) which turns out to be essentially the most stringent of the others - see Fig. 9. We observe that $m_{\chi} \sim 0.1 \mathrm{TeV}$ and $\langle\sigma v\rangle \sim 10^{-7} \mathrm{GeV}^{-2}\left[m_{\chi} \sim 1 \mathrm{TeV}\right.$ and $\langle\sigma v\rangle \sim 10^{-6} \mathrm{GeV}^{-2}$ ] for $\chi$ 's annihilating to $e^{+} e^{-}\left[\mu^{+} \mu^{-}\right]$. From the exposed in Table $3 \chi^{2}-\left.\chi^{2}\right|_{\min }$ 's, we deduce that all the requirements are met in a portion of the area favored at $99 \%$ c.l. [68\% c.l.] by the PAMELA and Fermi-LAT [ATIC] data for $\chi \chi \rightarrow \mu^{+} \mu^{-}$, whereas the mode $\chi \chi \rightarrow e^{+} e^{-}$can be excluded at $99 \%$ c.l. As regards the quality of the fits, from Tables 2 and 3, we can infer that the $\mu^{+} \mu^{-}$channel gives better fit to the PAMELA and Fermi-LAT data $\left(\chi^{2} /\right.$ d.o.f $\left.=33 / 31\right)$ than to the PAMELA and ATIC data $\left(\chi^{2} /\right.$ d.o.f $\left.=77 / 26\right)$.

From Table 3 we can also appreciate the importance of the non-SC in boosting $\Omega_{\chi} h^{2}$ to an acceptable level. Indeed, in this Table we display $\left.\Omega_{\chi} h^{2}\right|_{\text {SC }}$ for every allowed best-fit $\left(m_{\chi},\langle\sigma v\rangle\right)$. We observe that it lies much lower than the range of Eq. (1.2) in all cases, i.e., it is insufficient to account for the present CDM abundance in the universe. However, an appropriate adjustment (shown also in Table 3) of the parameters $c_{\chi \phi}$ and $T_{\mathrm{RH}}\left[b\right.$ and $\bar{H}_{\mathrm{I}}$ (with fixed $a=0.5$ and $T_{\mathrm{I}}=10^{9} \mathrm{GeV}$ ) $]$ for the LRS [QKS] - consistently with the restrictions of Sec. 2.2.2 [Sec. 2.3.2] - elevates adequately $\Omega_{\chi} h^{2}$ which can acquire the central experimental value in Eq. (1.2) for every best-fit point. In Table 3 we also expose the type of $\chi$ production for the LRS and the transition temperature to the $\mathrm{RD}$ era for the QKS. We remark that since the $\langle\sigma v\rangle$ 's required for $\chi$ 's annihilating to $e^{+} e^{-}$are lower than those required for the $\mu^{+} \mu^{-}$channel, non-TP dominates even for $T_{\mathrm{RH}}=0.1 \mathrm{GeV}$. Note that $T_{\mathrm{KR}} \leq 0.04 \mathrm{GeV}$ and the tracking behavior fails for $b=0$ in the case of the QKS.

\section{Conclusions}

We presented two non-standard cosmological scenaria which can increase the relic abundance of a WIMP $\chi, \Omega_{\chi} h^{2}$, w.r.t its value in the SC due to the generation of a background energy density steeper than the one of RD era. This increase is quantified by $\Delta \Omega_{\chi}$ defined in Eq. (3.10). According to the first scenario, termed LRS, a scalar field $\phi$ decays, reheating the universe to a reheating temperature lower than the freeze-out temperature of the WIMPs. According to the second scenario, termed QKS, a scalar field, $q$, rolls down its inverse power-low potential with a Hubble-induced mass term. In both cases our approach was both (i) purely numerical, integrating the relevant system of the differential equations (ii) semi-analytical, producing approximate relations for the evolution of the various energy densities of the cosmological background and the $\chi$-number density. We consider that the exposed semi-analytical findings - although do not provide quite accurate results in all cases - facilitate the understanding of the cosmological dynamics.

As regards the LRS, we recalled the dynamics of reheating and showed that $\Delta \Omega_{\chi}$ is affected by the two basic types of $\chi$ production which can be discriminated, depending whether non-TP dominates or equally contributes with TP. The first type is activated for very low $T_{\mathrm{RH}}$, low $N_{\chi}$ and is more or less independent of $\langle\sigma v\rangle$, whereas the latter case requires larger $T_{\mathrm{RH}}$ 's and $N_{\chi}$ 's and dependents on $\langle\sigma v\rangle$. In this last case, we remarked that a period of $\chi$ reannihilation can emerge. 


\begin{tabular}{|c|c|c|}
\hline & \multicolumn{2}{|c|}{ Fits To PAMELA AND ATIC DATA } \\
\cline { 2 - 3 } & $\chi \chi \rightarrow e^{+} e^{-}$ & $\chi \chi \rightarrow \mu^{+} \mu^{-}$ \\
\hline \hline$m_{\chi}(\mathrm{TeV})$ & 0.45 & 2 \\
$\langle\sigma v\rangle\left(\mathrm{GeV}^{-2}\right)$ & $1.98 \cdot 10^{-7}$ & $2.6 \cdot 10^{-6}$ \\
\hline$\chi^{2}-\left.\chi^{2}\right|_{\min }$ & 13.4 & 1 \\
\hline \hline$\left.\Omega_{\chi} h^{2}\right|_{\mathrm{SC}}$ & 0.0013 & 0.0001 \\
\hline \hline
\end{tabular}

Combinations of Parameters Yielding $\Omega_{\chi} h^{2}=0.11$ In The LRS

\begin{tabular}{|c|c|c|c||c|c|c|}
\hline$T_{\mathrm{RH}}(\mathrm{GeV})$ & 0.001 & 0.1 & 0.69 & 0.001 & 0.1 & 0.27 \\
$c_{\chi \phi}$ & $8.1 \cdot 10^{-5}$ & $1.3 \cdot 10^{-6}$ & 1 & $1.9 \cdot 10^{-5}$ & $9 \cdot 10^{-7}$ & 1 \\
\hline$\chi$-PRODUCTION & \multicolumn{2}{|c|}{ NON-TP } & NON-TP + TP & NON-TP & \multicolumn{2}{|c|}{ NON-TP + TP } \\
\hline \hline
\end{tabular}

Combinations of Parameters Yielding $\Omega_{\chi} h^{2}=0.11$

IN THE QKS FOR $a=0.5$ AND $T_{\mathrm{I}}=10^{9} \mathrm{GeV}$

\begin{tabular}{|c|c|c|c|c|c|}
\hline $\begin{array}{c}b \\
\bar{H}_{\mathrm{I}} / 10^{53} \\
\end{array}$ & $\begin{array}{c}0 \\
0.81\end{array}$ & $\begin{array}{c}0.2 \\
1.27\end{array}$ & $\begin{array}{c}0.32 \\
1\end{array}$ & $\begin{array}{c}0 \\
3.5\end{array}$ & $\begin{array}{l}0.08 \\
3.7\end{array}$ \\
\hline$T_{\mathrm{KR}}(\mathrm{GeV})$ & 0.06 & 0.03 & 0.038 & 0.017 & 0.005 \\
\hline & \multicolumn{5}{|c|}{ Fits to PAMELA ANd Fermi-LAT Data } \\
\hline & \multicolumn{3}{|c|}{$\chi \chi \rightarrow e^{+} e^{-}$} & \multicolumn{2}{|c|}{$\chi \chi \rightarrow \mu^{+} \mu^{-}$} \\
\hline $\begin{array}{c}m_{\chi}(\mathrm{TeV}) \\
\langle\sigma v\rangle\left(\mathrm{GeV}^{-2}\right)\end{array}$ & \multicolumn{3}{|c|}{$\begin{array}{c}0.383 \\
1.69 \cdot 10^{-7}\end{array}$} & \multicolumn{2}{|c|}{$\begin{array}{c}1.12 \\
1.44 \cdot 10^{-6} \\
\end{array}$} \\
\hline$\chi^{2}-\left.\chi^{2}\right|_{\text {min }}$ & \multicolumn{3}{|c|}{84} & \multicolumn{2}{|c|}{9} \\
\hline$\left.\Omega_{\chi} h^{2}\right|_{\mathrm{SC}}$ & \multicolumn{3}{|c|}{0.0015} & \multicolumn{2}{|c|}{0.00019} \\
\hline
\end{tabular}

Combinations of Parameters Yielding $\Omega_{\chi} h^{2}=0.11$ In The LRS

\begin{tabular}{|c|c|c|c||c|c|c|}
\hline$T_{\mathrm{RH}}(\mathrm{GeV})$ & 0.001 & 0.1 & 0.68 & 0.001 & 0.1 & 0.27 \\
$c_{\chi \phi}$ & $9.5 \cdot 10^{-5}$ & $1.5 \cdot 10^{-6}$ & 1 & $3.3 \cdot 10^{-5}$ & $1.4 \cdot 10^{-6}$ & 1 \\
\hline$\chi$-PRODUCTION & \multicolumn{2}{|c|}{ NON-TP } & NON-TP + TP & NON-TP & \multicolumn{2}{|c|}{ NON-TP + TP } \\
\hline \hline
\end{tabular}

\begin{tabular}{|c|c|c|c||c|c|c|}
\hline \multicolumn{7}{|c|}{ Combinations of Parameters Yielding $\Omega_{\chi} h^{2}=0.11$} \\
\hline \multicolumn{7}{|c|}{ In The QKS For $a=0.5$ And $T_{\mathrm{I}}=10^{9} \mathrm{GeV}$} \\
$\bar{H}_{\mathrm{I}} / 10^{53}$ & 0 & 0.2 & 0.32 & 0 & 0.08 & 0.18 \\
\hline$T_{\mathrm{KR}}(\mathrm{GeV})$ & 0.79 & 1.3 & 0.99 & 3.1 & 3.4 & 4.7 \\
\hline
\end{tabular}

TABLE 3: Best-fit $\left(m_{\chi},\langle\sigma v\rangle\right)$ 's for the combination of the PAMELA and ATIC or Fermi-LAT data and the various annihilation channels, consistently with all the imposed constraints. Shown are also the resulting $\chi^{2}-\left.\chi^{2}\right|_{\min }$ and $\Omega_{\chi} h^{2}$ in the $S C,\left.\Omega_{\chi} h^{2}\right|_{\mathrm{SC}}$, several combinations of $\left(b, \bar{H}_{\mathrm{I}}\right)$ 's $\left[\left(T_{\mathrm{RH}}, c_{\chi \phi}\right)\right.$ 's] leading to $\Omega_{\chi} h^{2} \simeq 0.11$ and the corresponding $T_{\mathrm{KR}}$ 's [types of $\chi$ production] within the QKS [LRS]. 
As regards the QKS, we verified that the included Hubble-induced mass term ensures the presence of a KD period, which is characterized by an oscillating evolution of $q$, and allows the quintessential energy density to join in time a tracker behavior, alleviating, thereby, the coincidence problem. Observational data originating from BBN, the present acceleration of the universe, the inflationary scale and the DE density parameter can be also met in a sizable fraction of the parameter space of the model. $\Delta \Omega_{\chi}$ crucially depends on the hierarchy between the freeze-out temperature and the temperature where the evolution of $q$ develops extrema.

Assuming that the WIMP annihilates primarily to $e^{+} e^{-}, \mu^{+} \mu^{-}$or $\tau^{+} \tau^{-}$we calculated the induced flux of $e^{ \pm}$-CRs and fit the current data of PAMELA, ATIC and Fermi LAT without invoking any ad-hoc boost factor. For simplicity, we did not include in our fits older experimental results, such as from PPB-BETS [70], or more uncertain ones, such as from H.E.S.S [71] (however, the latter data may be used for imposing an upper limit in the $m_{\chi}-\langle\sigma v\rangle$ plane [72]). Taking into account the strong bounds originating mostly from CMB, we concluded that the channel $\chi \chi \rightarrow \tau^{+} \tau^{-}$ can be excluded at $95 \%$ c.l. and: (i) large parts of the regions favored by PAMELA at $95 \%$ c.l. for the residual annihilation modes are ruled out; (ii) regions favored by PAMELA and ATIC or Fermi LAT at $99 \%$ c.l. for $\chi \chi \rightarrow e^{+} e^{-}$are excluded; (iii) only a part of the region favored by PAMELA and ATIC data at $95 \%$ c.l. for $\chi \chi \rightarrow \mu^{+} \mu^{-}$can be acceptable. For the latter annihilation channel we achieved our best fits to PAMELA and Fermi-LAT data with $m_{\chi} \sim 1 \mathrm{TeV}$ and $\langle\sigma v\rangle \sim 10^{-6} \mathrm{GeV}^{-2}$ which belong within the region individuated by these data-sets at $99 \%$ c.l. In all cases, the considered $m_{\chi}$ 's and $\langle\sigma v\rangle$ 's can yield the right amount of $\Omega_{\chi} h^{2}$ (entailed by the CDM considerations) by adjusting the parameters of the QKS or LRS. In both scenaria the required transition temperature to the conventional RD era turns out to be lower than about $0.7 \mathrm{GeV}$. In the case of the LRS an appreciable contribution of non-TP is also necessitated.

As for the prediction of any CDM signal, there are three sources of uncertainty in our investigation: the CDM distribution, the propagation of its annihilation products and the role of astrophysical backgrounds. In our analysis we used (i) the isothermal halo profile, to avoid troubles [31] with observations on $\gamma$-CRs; (ii) the MED propagation model, which provides the best fits to the combinations of the various data-sets [58] and (iii) commonly assumed background $e^{+}$and $e^{-}$ fluxes [48] normalized with the ATIC or Fermi-LAT data. The uncertainties above in conjunction with the very stringent constraints from $\mathrm{CMB}[33,34]$ may jeopardize the interpretation of the PAMELA and ATIC or Fermi-LAT anomalies through the $\chi$ annihilation. Therefore, the proposed scenaria can be probed in the near future, if a better understanding of the astrophysical uncertainties becomes available and/or more accurate experimental data are released.

Our proposal could be supplemented by the construction of a particle model (see, e.g., Ref. [58, 73]) with the appropriate couplings so that $\chi$ annihilates into $\mu^{+} \mu^{-}$with the desired $\langle\sigma v\rangle^{\prime}$ 's derived self-consistently with the (s)particle spectrum. In a such case, several phenomenological implications could be examined as in Ref. [15]. Let us finally mention that another class of non-standard cosmological scenaria $[8,23,47,74,75]$ can be generated considering modifications to the Friedmann equation due to corrections to the Einstein gravity. Constraining these possibilities (as in Ref. [76]) through the experimental results on $e^{ \pm}$-CRs would be another interesting issue. 


\section{ACKNOWLEDGMENTS}

This research was funded by the FP6 Marie Curie Excellence grant MEXT-CT-2004-014297. The author would like to thank K. Kohri for useful correspondence, A.B. Lahanas for valuable discussions and N.D. Vlachos for providing helpful software.

\section{REFERENCES}

[1] E. Komatsu et al. [WMAP Collaboration], Astrophys. J. Suppl. 180, 330 (2009) [arXiv: 0803.0547] http://lambda.gsfc.nasa.gov/product/map/dr2/parameters.cfm.

[2] For a review from the viewpoint of particle physics, see A.B. Lahanas, N.E. Mavromatos and D.V. Nanopoulos, Int. J. Mod. Phys. D 12, 1529 (2003) [hep-ph/0308251].

[3] K. Matchev, hep-ph/0402088; E.A. Baltz, astro-ph/0412170; G. LaZARIDEs, hep-ph/0601016; M. Taoso, G. Bertone and A. Masiero, J. Cosmol. Astropart. Phys. 03, 022 (2008) [arXiv: 0711.4996].

[4] G. Jungman, M. Kamionkowski And K. Griest, Phys. Rep. 267, 195 (1996) [hep-ph/9506380].

[5] G. Servant and T.M.P. Tait, Nucl. Phys. B650, 391 (2003) [hep-ph/0206071]; H.C. Cheng et al., Phys. Rev. Lett. 89, 211301 (2002) [hep-ph/0207125]; K. Agashe and G. Servant, Phys. Rev. Lett. 93, 231805 (2004) [hep-ph/0403143]; J.A.R. Cembranos et al., Phys. Rev. Lett. 90, 241301 (2003) [hep-ph/0302041].

[6] J. McDonald, Phys. Rev. Lett. 88, 091304 (2002) [hep-ph/0106249]; M. Cirelli, N. Fornengo and A. Strumia, Nucl. Phys. B753, 178 (2006) [hep-ph/0512090]; T. Asaka, K. Ishiwata and T. Moroi, Phys. Rev. D 73, 051301 (2006) [hep-ph/0512118]; D.G. Cerdeño, C. Muñoz and O. Seto, Phys. Rev. D 79, 023510 (2009) [arXiv: 0807. 3029]; F. Deppisch and A. Pilaftsis, J. High Energy Phys. 10, 080 (2008) [arXiv: 0808. 0490].

[7] H. Goldberg, Phys. Rev. Lett. 50, 1419 (1983); J.R. Eluis et al., Nucl. Phys. B238, 453 (1984).

[8] M. Kamionkowski and M.S. Turner, Phys. Rev. D 42, 3310 (1990); C. PALlis, "The Identification of Dark Matter", pp. 602-608 [hep-ph/0610433].

[9] T. Moroi and L. Randall, Nucl. Phys. B570, 455 (2000) [hep-ph/9906527]; R. Allahverdi And M. Drees, Phys. Rev. Lett. 89, 091302 (2002) [hep-ph/0203118].

[10] J. McDonald, Phys. Rev. D 43, 1063 (1991); T. Nagano and M. Yamaguchi, Phys. Lett. B 438, 267 (1998) [hep-ph/9805204]; G.F. Giudice, E.W. Kolb And A. Riotto, Phys. Rev. D 64, 023508 (2001) [hep-ph/0005123]; N. Fornengo, A. Riotto and S. Scopel, Phys. Rev. D 67, 023514 (2003) [hep-ph/0208072].

[11] C. Pallis, Astropart. Phys. 21, 689 (2004) [hep-ph/0402033]; C. Pallis, Nucl. Phys. B751, 129 (2006) [hep-ph/0510234].

[12] G.B. Gelmini and P. Gondolo, Phys. Rev. D 74, 023510 (2006) [hep-ph/0602230]; G. Gelmini, P. Gondolo, A. Soldatenko and C.E. Yaguna, Phys. Rev. D 74, 083514 (2006) [hep-ph/0605016].

[13] M. Nagai and K. Nakayama, Phys. Rev. D 78, 063540 (2008) [arXiv:0807.1634]; B. Dutta, L. Leblond and K. Sinha, Phys. Rev. D 80, 035014 (2009) [arXiv: 0904 . 3773]; J. McDonald, Phys. Rev. Lett. 103, 151301 (2009) [arXiv:0904.0969].

[14] P. Salati, Phys. Lett. B 571, 121 (2003) [astro-ph/0207396].

[15] S. Profumo and P. Ullio, J. Cosmol. Astropart. Phys. 11, 006 (2003) [hep-ph/0309220]; D.J.H. Chung et al., J. High Energy Phys. 10, 016 (2007) [arXiv: 0706.2375]. 
[16] C. Pallis, J. Cosmol. Astropart. Phys. 10, 015 (2005) [hep-ph/0503080];

M.E. Gómez et al., J. Cosmol. Astropart. Phys. 01, 027 (2009) [arXiv: 0809. 1859];

M.E. Gómez et al., AIP Conf. Proc. 1115, 157 (2009) [arXiv: 0809. 1982].

[17] B. Spokoiny, Phys. Lett. B 315, 40 (1993) [gr-qc/9306008];

M. Joyce, Phys. Rev. D 55, 1875 (1997) [hep-ph/9606223];

P.G. Ferreira And M. Joyce, Phys. Rev. D 58, 023503 (1998) [astro-ph/9711102].

[18] P.J. Peebles and A. Vilenkin, Phys. Rev. D 59, 063505 (1999) [astro-ph/9810509];

M. Yahiro et al., Phys. Rev. D 65, 063502 (2002) [astro-ph/0106349];

K. Dimopoulos and J.W. Valle, Astropart. Phys. 18, 287 (2002) [astro-ph/0111417];

K. Dimopoulos, Phys. Rev. D 68, 123506 (2003) [astro-ph/0212264];

I.P. Neupane, Class. Quant. Grav. 25, 125013 (2008) [arXiv: 0706.2654];

M. Bastero-Gil, A. Berera, B.M. Jackson and A. Taylor, Phys. Lett. B 678, 157 (2009) [arXiv: 0905.2937].

[19] D.J.H. Chung, L.L. Everett and K.T. Matchev, Phys. Rev. D 76, 103530 (2007) [arXiv: 0704.3285];

G. Barenboim and J.D. Lykken, J. High Energy Phys. 12, 005 (2006) [hep-ph/0608265];

G. Barenboim And J.D. Lykken, J. High Energy Phys. 10, 032 (2007) [arXiv: 0707 . 3999].

[20] A. Masiero, M. Pietroni and F. Rosati, Phys. Rev. D 61, 023504 (2000) [hep-ph/9905346];

F. Rosati, Phys. Lett. B 570, 5 (2003) [hep-ph/0302159].

[21] S. Lola, C. Pallis and E. Tzelati, J. Cosmol. Astropart. Phys. 11, 017 (2009) [arXiv: 0907. 2941].

[22] P. Binetrui, Int. J. Theor. Phys. 39, 1859 (2000) [hep-ph/0005037];

E.J. Copeland et al., Int. J. Mod. Phys. D 15, 1936 (2006) [hep-th/0603057].

[23] A.A.E. Zant, S. Khalil And H. OKada, arXiv:0903.5083;

W.L. Guo and X. Zhang, Phys. Rev. D 79, 115023 (2009) [arXiv:0904.2451].

[24] O. Adriani et al. [PAMELA Collaboration], Nature 458, 607 (2009) [arXiv: 0810 . 4995].

[25] J. Chang et al. [ATIC Collaboration], Nature 456, 362 (2008).

[26] M. Ibe, H. Murayama and T.T. Yanagida, Phys. Rev. D 79, 095009 (2009) [arXiv:0812.0072]; W.L. Guo and Y.L. Wu, Phys. Rev. D 79, 055012 (2009) [arXiv: 0901. 1450].

[27] J. Hisano, S. Matsumoto, M.M. Nojiri and O. Saito, Phys. Rev. D 71, 063528 (2005) [hep-ph/0412403]; N. Arkani-Hamed et al., Phys. Rev. D 79, 015014 (2009) [arXiv:0810.0713];

M. Lattanzi and J.I. Silk, Phys. Rev. D 79, 083523 (2009) [arXiv: 0812 .0360];

J.D. March-Russell and S.M. West, Phys. Lett. B 676, 133 (2009) [arXiv: 0812.0559];

X.G. He, arXiv:0908.2908.

[28] D. Hooper et al., J. Cosmol. Astropart. Phys. 01, 025 (2009) [arXiv:0810.1527];

H. Yuksel, M. D. Kistler and T. Stanev, Phys. Rev. Lett. 103, 051101 (2009) [arXiv:0810.2784].

[29] S.W. Barwick et al. [HEAT Collaboration], Astrophys. J.482, L191 (1997) [astro-ph/9703192]; M. Aguilar et al. [AMS-01 Collaboration], Phys. Lett. B 646, 145 (2007) [astro-ph/0703154].

[30] A.A. Abdo et al. [The Fermi-LAT Collaboration], Phys. Rev. Lett. 102, 181101 (2009) [arXiv:0905.0025].

[31] G. Bertone, M. Cirelli, A. Strumia and M. Taoso, J. Cosmol. Astropart. Phys. 03, 009 (2009) [arXiv: 0811. 3744].

[32] N. Padmanabhan and D.P. Finkbeiner, Phys. Rev. D 72, 023508 (2005) [astro-ph/0503486];

L. Zhang, X.L. Chen, Y.A. Lei and Z.G. Si, Phys. Rev. D 74, 103519 (2006) [astro-ph/0603425].

[33] S. Galli, F. Iocco, G. Bertone and A. Melchiorri, Phys. Rev. D 80, 023505 (2009) [arXiv:0905.0003]; T.R. Slatyer, N. Padmanabhan and D.P. Finkbeiner, Phys. Rev. D 80, 043526 (2009) [arXiv: 0906. 1197].

[34] G. Huetsi, A. Hektor and M. Raidal, Astron. Astrophys. 505, 999 (2009) [arXiv: 0906.4550];

M. Cirelli, F. Iocco and P. Panci, J. Cosmol. Astropart. Phys. 10, 009 (2009) [arXiv: 0907. 0719];

T. KanZaKi, M. KaWASAKI AND K. NaKayama, arXiv:0907.3985. 
[35] M. Dine, L. Randall and S.D. Thomas, Phys. Rev. Lett. 75, 398 (1995) [hep-ph/9503303]; D.J.H. Chung, L.L. Everett and A. Riotto, Phys. Lett. B 556, 61 (2003) [hep-ph/0210427].

[36] S.D.H. Hsu and B. Murray, Phys. Lett. B 595, 16 (2004) [astro-ph/0402541]; D.J. Liu and X.Z. Li, Phys. Lett. B 611, 8 (2005) [astro-ph/0501596]; W.Z. Liu and D.J. LiU, arXiv:0803.4039.

[37] P.J. Steinhardt, L. Wang and I. Zlatev, Phys. Rev. Lett. 82, 896 (1999) [astro-ph/9807002]; ibid., Phys. Rev. D 59, 123504 (1999) [astro-ph/9812313].

[38] B. Ratra and P.J.E. Peebles, Phys. Rev. D 37, 3406 (1988); A.R. Liddle AND R.J. Scherrer, Phys. Rev. D 59, 023509 (1999) [astro-ph/9809272]; J.P. Uzan, Phys. Rev. D 59, 123510 (1999) [gr-qc/9903004].

[39] G. Bélanger et al., Comput. Phys. Commun. 149, 103 (2002) [hep-ph/0112278]; P. Gondolo et al., J. Cosmol. Astropart. Phys. 07, 008 (2004) [astro-ph/0406204].

[40] R.H. Cyвurt et al., Astropart. Phys. 23, 313 (2005) [astro-ph/0408033].

[41] S. Davidson and S. Sarkar, J. High Energy Phys. 11, 012 (2000) [hep-ph/0009078].

[42] M. Endo et al., Phys. Rev. Lett. 96, 211301 (2006) [hep-ph/0602061]; S. Nakamura and M. Yamaguchi, Phys. Lett. B 638, 389 (2006) [hep-ph/0602081].

[43] M. Tegmark et al., Phys. Rev. D 69, 103501 (2004) [astro-ph/0310723]; A.G. Riess et al., Astrophys. J. 607, 665 (2004) [astro-ph/0402512].

[44] P. Binetruy, Phys. Rev. D 60, 063502 (1999) [hep-ph/9810553]; P. Binetruy, Supersymmetry, Oxford University Press (2006).

[45] C. Baccigalupi et al., Phys. Rev. D 65, 063520 (2002) [astro-ph/0109097].

[46] G. Lazarides, R.K. Schaefer, and Q. Shafi, Phys. Rev. D 56, 1324 (1997) [hep-ph/9608256].

[47] R. Catena et al., Phys. Rev. D 70, 063519 (2004) [astro-ph/0403614]; R. Catena et al., J. High Energy Phys. 10, 003 (2008) [arXiv: 0712.3173].

[48] E.A. Baltz and J. Edsjo, Phys. Rev. D 59, 023511 (1999) [astro-ph/9808243].

[49] J. Hisano, S. Matsumoto, O. Saito and M. Senami, Phys. Rev. D 73, 055004 (2006) [hep-ph/0511118]; T. Delahaye et al., Phys. Rev. D 77, 063527 (2008) [arXiv:0712.2312].

[50] M. Cirelli, R. Franceschini and A. Strumia, Nucl. Phys. B800, 204 (2008) [arXiv: 0802 . 3378].

[51] O. Adriani et al. [PAMElA Collaboration], Phys. Rev. D 102, 051101 (2009) [arXiv: 0810.4994].

[52] P. Grajek, G. Kane, D. Phalen, A. Pierce and S. Watson, Phys. Rev. D 79, 043506 (2009) [arXiv: 0812 .4555]; G. Kane, R. Lu and S. Watson, Phys. Lett. B 681, 151 (2009) [arXiv: 0906.4765].

[53] M.E. Peskin and D.V. Schroeder, An Introduction to Quantum Field Theory, Addison-Wesley, Reading, (1995).

[54] I.Z. Rothstein, T. Schwetz and J. Zupan, J. Cosmol. Astropart. Phys. 07, 018 (2009) [arXiv: 0903. 3116 ].

[55] Y. Bai, M. Carena and J. Lykken, Phys. Rev. D 80, 055004 (2009) [arXiv: 0905.2964].

[56] T. Suostrand, S. Mrenna and P. Skands, J. High Energy Phys. 05, 026 (2006) [hep-ph/0603175].

[57] L. Bergstrom et al., Phys. Rev. Lett. 94, 131301 (2005) [astro-ph/0410359];

A. Birkedal, K.T. Matchev, M. Perelstein and A. Spray, hep-ph/0507194.

[58] K. Cheung, P.Y. Tseng and T.C. Yuan, Phys. Lett. B 678, 293 (2009) [arXiv: 0902 .4035].

[59] J.N. Bahcall and R.M. Soneira, Astrophys. J. Suppl. 44, 73 (1980).

[60] J.F. Navarro, C.S. Frenk and S.D. White, Astrophys. J. 462, 563 (1996) [astro-ph/9508025].

[61] J. Hisano et al., Phys. Rev. D 79, 083522 (2009) [arXiv: 0901.3582]. 
[62] K. Ishiwata, S. Matsumoto and T. Moroi, J. High Energy Phys. 05, 110 (2009) [arXiv: 0903.0242].

[63] Ilias Cholis et al., Phys. Rev. D 80, 123518 (2009) [arXiv: 0811.3641];

P. Meadea, M. Papucci, A. Strumia and T. Volansky, arXiv:0905.0480.

[64] M.H. Reno and D. Seckel, Phys. Rev. D 37, 3441 (1988);

J.A. Frieman, E.W. Kolb and M.S. Turner, Phys. Rev. D 41, 3080 (1990);

K. Jedamzik, Phys. Rev. D 70, 083510 (2004) [astro-ph/0405583].

[65] K. Griest and M. Kamionkowski, Phys. Rev. Lett. 64, 615 (1990); L. Hui, Phys. Rev. Lett. 86, 3467 (2001).

[66] F. Aharonian et al. [H.E.S.S Collaboration], Astropart. Phys. 29, 55 (2008) [arXiv:0711.2369].

[67] P. Sreekumar et al. [EGRET Collaboration], Astrophys. J. 494, 523 (1998) [astro-ph/9709257]; A.W. Strong, I.V. Moskalenko and O. Reimer, Astrophys. J. 613, 962 (2004) [astro-ph/0406254].

[68] E. Borriello, A. Cuoco and G. Miele, Astrophys. J. 699, 59 (2009) [arXiv:0903. 1852];

M. Cirelli and P. Panci, Nucl. Phys. B821, 399 (2009) [arXiv: 0904 . 3830];

S. Profumo and T.E. Jeltema, J. Cosmol. Astropart. Phys. 07, 020 (2009) [arXiv:0906.0001].

[69] J. Hisano et al., Phys. Rev. D 79, 043516 (2009) [arXiv: 0812.0219];

J. Liu, P.f. Yin and S.h. Zhu, Phys. Rev. D 79, 063522 (2009) [arXiv:0812.0964].

[70] S. Toril et al. [PPB-BETS Collaboration], arXiv:0809.0760.

[71] F. Aharonian et al. [H.E.S.S. Collaboration], Phys. Rev. Lett. 101, 261104 (2008) [arXiv: 0811. 3894]; F. Aharonian et al. [H.E.S.S. Collaboration], arXiv:0905.0105.

[72] L. Bergstrom, J. Edsjo and G. Zaharijas, Phys. Rev. Lett. 103, 031103 (2009) [arXiv:0905.0333].

[73] M. Cirelli, M. Kadastik, M. Raidal and A. Strumia, Nucl. Phys. B813, 1 (2009) [arXiv: 0809. 2409]; D. Feldman, Z. Liu and P. Nath, Phys. Rev. D 79, 063509 (2009) [arXiv: 0810. 5762];

R. Allahverdi et al., Phys. Rev. D 79, 075005 (2009) [arXiv: 0812 .2196];

I. Gogoladze, R. Khalid, Q. Shafi and H. Yuksel, Phys. Rev. D 79, 055019 (2009) [arXiv:0901.0923];

C. Balazs, N. Sahu and A. Mazumdar, J. Cosmol. Astropart. Phys. 07, 039 (2009) [arXiv: 0905.4302];

X.J. Bi et al., Phys. Rev. D 80, 103502 (2009) [arXiv:0905.1253];

F. Chen, J.M. Cline and A.R. Frey, Phys. Rev. D 80, 083516 (2009) [arXiv: 0907.4746];

D. Feldman, Z. Liu, P. Nath and B.D. Nelson, Phys. Rev. D 80, 075001 (2009) [arXiv:0907. 5392].

[74] A.B. Lahanas, N.E. Mavromatos and D.V. Nanopoulos, Phys. Lett. B 649, 83 (2007) [hep-ph/0612152]; M. Drees, H. Iminniyaz and M. Kakizaki, Phys. Rev. D 76, 103524 (2007) [arXiv: 0704 . 1590].

[75] N. OKada and O. Seto, Phys. Rev. D 70, 083531 (2004) [hep-ph/0407092]; T. Ninei, N. OKada and O. Seto, Phys. Rev. D 71, 063535 (2005) [hep-ph/0409219].

[76] R. Catena, N. Fornengo, M. Pato, L. Pieri and A. Masiero, arXiv: 0912 . 4421. 